\title{
Intravascular hemolysis activates complement via cell-free heme and heme-loaded microvesicles
}

Nicolas S. Merle, ${ }^{1,2,3}$ Anne Grunenwald, ${ }^{1,3,4}$ Helena Rajaratnam, ${ }^{1,5}$ Viviane Gnemmi, ${ }^{4}$ Marie Frimat, ${ }^{6,7}$ Marie-Lucile Figueres, ${ }^{1,2,3}$ Samantha Knockaert, ${ }^{1,2,3}$ Sanah Bouzekri, ${ }^{1,3}$ Dominique Charue, ${ }^{3,8}$ Remi Noe, ${ }^{1,9}$ Tania Robe-Rybkine, ${ }^{1,2,3}$ Marie Le-Hoang, ${ }^{3,8}$ Nathan Brinkman, ${ }^{10}$ Thomas Gentinetta, $^{11}$ Monika Edler, ${ }^{11}$ Sara Petrillo, ${ }^{12}$ Emanuela Tolosano, ${ }^{12}$ Sylvia Miescher, ${ }^{11}$ Sylvain Le Jeune, ${ }^{13}$ Pascal Houillier, ${ }^{1,2,3}$ Sophie Chauvet, ${ }^{1,2,3,14}$ Marion Rabant, ${ }^{15}$ Jordan D. Dimitrov, ${ }^{1,2,3}$ Veronique Fremeaux-Bacchi, ${ }^{1,2,3,16}$ Olivier P. Blanc-Brude,, ${ }^{3,8}$ and Lubka T. Roumenina, ${ }^{1,2,3}$ IINSERM, UMRS 1138, Centre de Recherche des Cordeliers, Paris, France. ${ }^{2}$ Sorbonne Universités, Université Pierre et Marie Curie - Paris 06, Paris France. ${ }^{3}$ Université Paris Descartes, Sorbonne Paris Cité, Paris, France. ${ }^{4}$ Université Lille, INSERM, CHRU Lille, Service de pathologie, UMRS 1172, Jean-Pierre Aubert Research Center, Lille, France. ${ }^{5}$ SupBiotech Paris, Villejuif, France. ${ }^{6} I N S E R M$, UMR 995, Lille, France. ${ }^{7}$ CHRU Lille, Service de néphrologie, Lille, France. ${ }^{8}$ Paris Center for Cardiovascular Research, INSERM UMRS 970, Paris, France. ${ }^{9}$ Ecole Pratique des Hautes Études, Paris, France. ${ }^{10} \mathrm{CSL}$ Behring, R\&D, Kankakee, Illinois, USA. "'CSL Behring, Research, Bern, Switzerland. ${ }^{2}$ Department Molecular Biotechnology and Health Sciences, Molecular Biotechnology Center, University of Torino, Torino, Italy. ${ }^{13}$ Assistance Publique - Hôpitaux de Paris, Service de Médecine Interne, Hôpital Avicenne, Bobigny, France. ${ }^{14}$ Assistance Publique - Hôpitaux de Paris, Service de Néphrologie, Hôpital Européen Georges Pompidou, Paris, France. ${ }^{15}$ Assistance Publique - Hôpitaux de Paris, Service de Pathologie, Hôpital Necker Enfants Malades, Paris, France. ${ }^{16}$ Assistance Publique - Hôpitaux de Paris, Service d'Immunologie Biologique, Hôpital Européen Georges Pompidou, Paris, France.

In hemolytic diseases, such as sickle cell disease (SCD), intravascular hemolysis results in the release of hemoglobin, heme, and heme-loaded membrane microvesicles in the bloodstream. Intravascular hemolysis is thus associated with inflammation and organ injury. Complement system can be activated by heme in vitro. We investigated the mechanisms by which hemolysis and red blood cell (RBC) degradation products trigger complement activation in vivo. In kidney biopsies of SCD nephropathy patients and a mouse model with SCD, we detected tissue deposits of complement C3 and C5b-9. Moreover, drug-induced intravascular hemolysis or injection of heme or hemoglobin in mice triggered C3 deposition, primarily in kidneys. Renal injury markers (Kim-1, NGAL) were attenuated in $\mathrm{C}^{-/-}$hemolytic mice. RBC degradation products, such as heme-loaded microvesicles and heme, induced alternative and terminal complement pathway activation in sera and on endothelial surfaces, in contrast to hemoglobin. Heme triggered rapid P selectin, $C 3 a R$, and $\mathrm{C} 5 \mathrm{aR}$ expression and downregulated CD46 on endothelial cells. Importantly, complement deposition was attenuated in vivo and in vitro by heme scavenger hemopexin. In conclusion, we demonstrate that intravascular hemolysis triggers complement activation in vivo, encouraging further studies on its role in SCD nephropathy. Conversely, heme inhibition using hemopexin may provide a novel therapeutic opportunity to limit complement activation in hemolytic diseases.

Conflict of interest: NB, TG, ME, and SM are employees of CSL Behring. LTR receives research funding from $C S L$ Behring.

Submitted: August 18, 2017

Accepted: May 8, 2018

Published: June 21, 2018

\section{Reference information:}

JCI Insight. 2018;3(12):e96910.

https://doi.org/10.1172/jci.

insight. 96910

\section{Introduction}

Intravascular hemolysis is a pathological event, leading to red blood cell (RBC) degradation and liberation of hemoglobin $(\mathrm{Hb})$ in the bloodstream. Upon depletion of the $\mathrm{Hb}$-scavenging protein haptoglobin $(\mathrm{Hp})$, $\mathrm{Hb}$ oxidation occurs and heme is released from the globin chains and is rapidly bound by hemopexin ( $\mathrm{Hx})$ (1). Upon Hx saturation, the remaining labile cell-free and Hx-free heme mediates oxidative stress, vascular damage, and inflammation (2).

Heme has been described as a key player in the pathogenesis of various diseases, such as malaria, sepsis, transfusion complications, and sickle cell disease (SCD) (2). SCD is a severe genetic disease and a major 
public health concern in many countries, affecting around 6 million individuals on all inhabited continents (3). In SCD heme participates in vaso-occlusive crises (VOC), endothelial cell (EC) toxicity, and ROS generation (4-6). SCD patients display high levels of heme-loaded RBC microvesicles (MVs), which distribute heme to tissues and contribute to EC damage and VOCs $(5,7)$. Heme acts as a damage-associated molecular pattern and triggers activation of monocytes (8) and ECs (4-6). In vitro studies suggest that heme activates the innate immune complement system in sera and on RBC surfaces (2, 9-12). In addition, triggering complement by heme causes an inflammatory reaction, leading to the release of anaphylatoxins $\mathrm{C} 3 \mathrm{a}$ and C5a and the formation of the membrane attack complex C5b-9 on EC surfaces (12). Clinical observations showed a significant increase of pathological markers of complement activation in SCD sera, especially during painful VOCs (13-15), as well as increased levels of surface-bound C3 fragments on RBC $(13,16)$.

Renal damage is a complication of intravascular hemolysis in many diseases, including SCD (3). Moreover, the kidney is particularly susceptible to complement attack (9). In SCD nephropathy, the exact mechanism and pathophysiological relevance of complement activation is not established, despite some early reports mentioning C3 fragment deposition in kidneys in humans and in a mouse model of SCD (17-19).

Here, we demonstrate that complement activation proceeds all the way through the terminal pathway during SCD nephropathy in humans as well as in kidneys of two SCD mouse models. Moreover, hemolysis-related products - Hb-derived heme and heme-loaded RBC-derived MVs - activated complement in vitro, in sera and on cultured ECs, as well as in vivo, in a mouse model of intravascular hemolysis. In vivo and in vitro, the deposition of the $\mathrm{C} 3$ fragments was antagonized by pretreatment with $\mathrm{Hx}$.

\section{Results}

Cohort of SCD nephropathy. On light microscopy, the predominant pattern of the observed glomerular disease was specific SCD glomerulopathy $(n=5)$, focal segmental glomerulosclerosis $(n=3)$, membranoproliferative glomerulonephritis $(n=3)$, and thrombotic microangiopathy (TMA) $(n=2)$ (Table 1). In 10 of 13 patients, glomerular lesions were associated with chronic tubulointerstitial lesions with different degrees of interstitial fibrosis and tubular atrophy. Three patients had tubular casts and six patients showed inflammatory cell infiltrate (macrophages). Perls' staining revealed hemosiderosis near or in renal tubules in 7 of 8 tested patients. Chronic vascular lesions were observed in 10 of 13 patients, resulting in hyaline arteriosclerosis in most of patients. One patient had TMA lesions involving extraglomerular small vessels. By immunofluorescence, C3 staining was positive in 9 of 11 patients in glomeruli (capillary walls and/or mesangium) and/or in vessels. Polyclonal immunoglobulin staining was positive in glomeruli of 4 of 10 patients.

Complement deposition is present in kidneys of patients and mice with SCD. C3 staining was positive in 9 of 11 (82\%) SCD nephropathy patient biopsies in glomeruli (capillary walls and/or mesangium) and/or vessels (Figure 1A and Table 1) and was absent from normal kidneys. Most (2 of 3) tested SCD biopsies were positive for C9 staining (Figure 1B). A graft rejection biopsy is shown as positive control for C3 and C9 staining. Polyclonal immunoglobulin staining was positive in glomeruli of 5 of 11 (45\%) patients (Table 1).

A similar pattern of increased C3 and C5b-9 deposition was detected in transgenic HbSS (Figure 1C) as well as SAD mouse kidneys (Figure 1D) compared with HbAA and WT control littermates, respectively. C3 and C5b-9 deposition was mostly intraglomerular and partly peritubular (Figure 1, C and D). Moreover, complement activation partially colocalized with EC marker CD31 in glomeruli (Figure 1E). Both SAD and HbSS mice were characterized by increased plasma levels of RBC MVs and cell-free heme (Supplemental Figure 1; supplemental material available online with this article; https://doi.org/10.1172/ jci.insight.96910DS1).

Description of a model of intravascular hemolysis. SCD nephropathy, where we detected C3 deposits (Figure $1 \mathrm{~A})$, is a chronic disease. We used a well-characterized model of drug-induced intravascular hemolysis in mice $(9,10)$ in order to determine whether the complement deposits observed in the kidneys of sickle patients and sickle mice were directly related to hemolysis.

Widespread hemolysis was evident in mice treated with phenylhydrazine (PHZ) from the darker color of the urine and organs (data not shown), markedly decreased hematocrits, increased cell-free plasma $\mathrm{Hb}$, and RBC MV and heme levels at 24 hours after injection (Supplemental Figure 2, A-D). Intravascular hemolysis was already evident 6 hours after injection (Supplemental Figure 2D). About 15\% of the total plasma heme was contained in the MVs, as measured by the decrease in the absorbance of plasma before and after ultracentrifugation to remove the MVs (Supplemental Figure 2E). The plasma concentration of 
Table 1. Description of the renal biopsies of patients with SCD nephropathy

\begin{tabular}{|c|c|c|c|c|c|c|c|c|c|c|c|c|c|}
\hline Patient no. & 1 & 2 & 3 & 4 & 5 & 6 & 7 & 8 & 9 & 10 & 11 & 12 & 13 \\
\hline \multicolumn{14}{|l|}{ Light microscopy } \\
\hline No. glomeruli & 17 & 15 & 15 & 25 & na & 18 & 7 & 22 & 22 & 9 & 12 & 10 & 36 \\
\hline $\begin{array}{l}\text { No. sclerotic glomeruli } \\
(\%)\end{array}$ & $0(0)$ & $5(33)$ & $10(66)$ & $8(32)$ & na & $4(22)$ & $3(43)$ & $5(23)$ & $6(27)$ & $2(22)$ & $3(25)$ & $1(10)$ & $6(17)$ \\
\hline \multicolumn{14}{|l|}{ Glomerular lesions } \\
\hline $\begin{array}{l}\text { Glomerular } \\
\text { hypertrophy }\end{array}$ & ++ & ++ & + & ++ & +++ & + & + & - & na & ++ & ++ & ++ & + \\
\hline $\begin{array}{l}\text { Mesangial } \\
\text { hypertrophy }\end{array}$ & + & ++ & + & ++ & - & ++ & +++ & - & ++ & ++ & ++ & + & + \\
\hline $\begin{array}{l}\text { Mesangial } \\
\text { proliferation }\end{array}$ & & - & ++ & + & - & + & - & - & - & - & - & - & - \\
\hline Duplication of GBM & - & ++ & - & ++ & + & +++ & + & ++ & - & + & + & - & - \\
\hline FSGS & - & - & ++ & - & + & + & + & + & ++ & - & + & + & ++ \\
\hline Capillaries distention & + & na & - & - & + & + & - & - & - & + & ++ & ++ & na \\
\hline $\begin{array}{l}\text { Intracapillary } \\
\text { thrombus }\end{array}$ & - & - & - & - & - & + & - & - & - & + & - & + & + \\
\hline \multicolumn{14}{|l|}{$\begin{array}{l}\text { Tubulointerstitial } \\
\text { lesions }\end{array}$} \\
\hline Interstitial fibrosis & - & +++ & +++ & +++ & - & +++ & - & +++ & ++ & + & +++ & ++ & +++ \\
\hline Tubular atrophy & - & +++ & ++ & +++ & - & +++ & - & +++ & ++ & + & +++ & ++ & +++ \\
\hline Cellular infiltration & - & - & - & - & - & - & - & + & + & + & + & + & +++ \\
\hline Casts & - & + & + & - & - & - & - & - & - & - & + & - & na \\
\hline Vascular lesions & HA & $\mathrm{HA}$ & $\mathrm{HA}$ & $\mathrm{HA}$ & NS & NS, HA & $\mathrm{HA}$ & NS, HA & HA & TMA & - & - & NS \\
\hline Perls' staining & na & + & na & na & + & na & - & na & + & + & + & + & + \\
\hline \multicolumn{14}{|l|}{ Immunofluorescence } \\
\hline C3 & na & CW, Mes & Mes & Vessel & - & na & Mes & Vessel & Mes & $\begin{array}{c}\text { CW, } \\
\text { vessel }\end{array}$ & Mes, CW & - & Mes \\
\hline Fibrin & na & - & - & CW & - & na & - & - & - & - & - & - & - \\
\hline $\lg$ & na & CW & Mes & $\mathrm{CW}$ & - & na & $\mathrm{CW}$ & - & Mes & - & - & - & - \\
\hline $\begin{array}{l}\text { Predominant } \\
\text { histological pattern }\end{array}$ & SCDG & MPGN & FSGS & SCDG & SCDG & MPGN & MPGN & TMA & FSGS & TMA & SCDG & SCDG & FSGS \\
\hline
\end{tabular}

CW, capillary wall; FSCS, focal segmental glomerulosclerosis; GBM, glomerular basement membrane; HA, hyaline arteriosclerosis; MPGN, membranoproliferative glomerulonephritis, Mes, mesangium; na, not available; NS, nephrosclerosis; SCDG, sickle cell disease glomerulopathy; TMA, thrombotic microangiopathy lesions. Lesions were graded using the following scale: -, no lesion; +, rare or few lesions; ++, moderate lesions; and +++, severe lesions.

endogenous $\mathrm{Hx}$ was about $0.15 \mathrm{mg} / \mathrm{ml}$ in resting mice. Six hours after PHZ injection, Hx levels were substantially decreased, but they increased again 24 hours after treatment, suggesting consumption by hemolysis followed by de novo expression (Supplemental Figure 2F). Conversely, treatment with exogenous human sequence $\mathrm{Hx}$ did not modulate endogenous mouse Hx levels in plasma (Supplemental Figure 2F). Human $\mathrm{Hx}$ was found at concentrations of up to $0.4 \mathrm{mg} / \mathrm{ml}$ (over 2-fold above basal levels of endogenous Hx) 6 hours after Hx injection and remained detectable for at least 24 hours (Supplemental Figure 2G).

Immunohistochemistry of kidney sections revealed no HO-1 expression in PBS- and Hx-treated mice (Supplemental Figure 3A). Pharmacological induction of hemolysis with PHZ triggered HO-1 expression within 24 hours. This was not modified by Hx supplementation (Supplemental Figure 3A). Perls' Prussian blue staining for hemosiderin, a hallmark of hemolysis, became evident 24 hours after PHZ injection (Supplemental Figure 3B). The analysis of kidney sections from PBS- and PHZ-treated WT mice revealed no gross modification of histology (hematoxylin and eosin staining) and glomerulus size by light microscopy (Supplemental Figure 3, C and D).

Intravascular hemolysis induced heme-dependent complement deposits in kidneys and on endothelium and complement-mediated renal injury. We detected a significant increase in C3 activation fragment deposition in renal glomeruli within 6 hours (Figure 2A) after inducing intravascular hemolysis with PHZ; this increase was maintained for at least 24 hours (Figure 2B). This was indeed concomitant with 
A

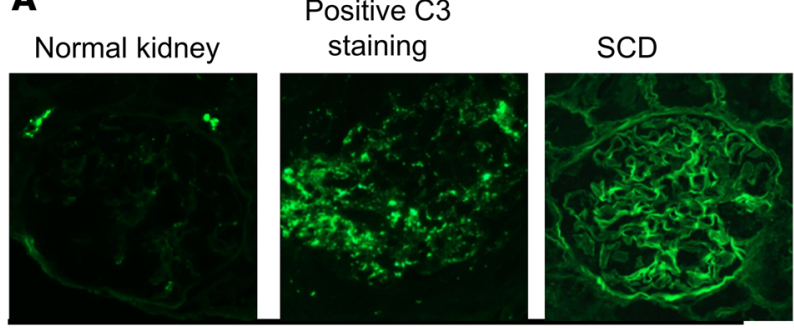

C3 fragment deposition
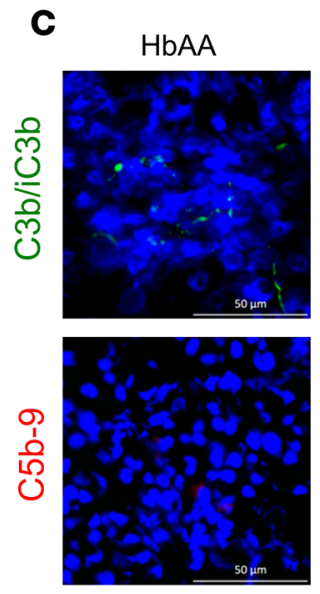

E
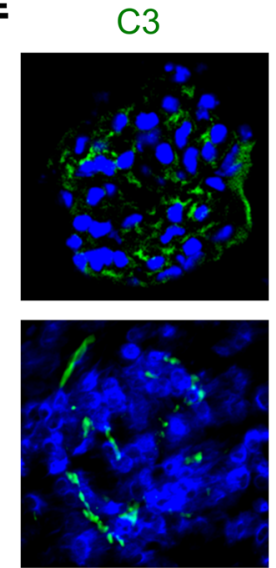

HbSS
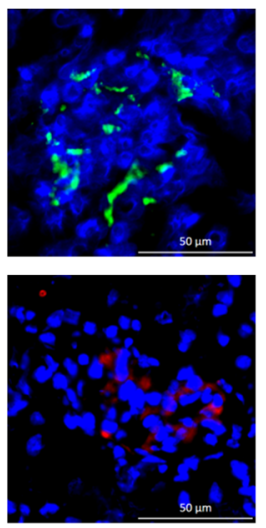

CD31
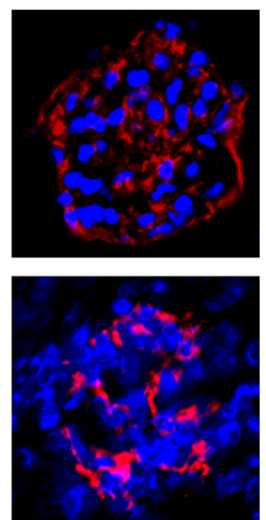

B

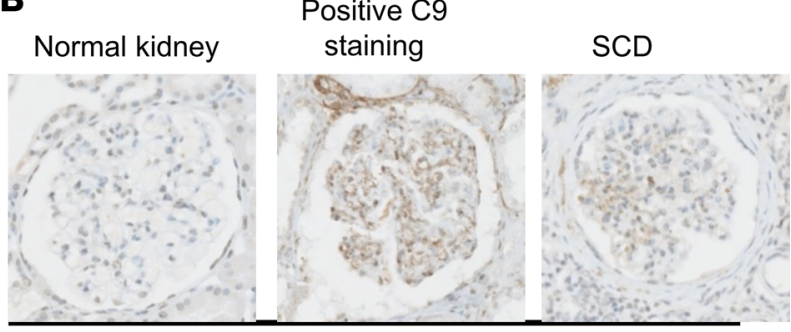

C9 deposition

D
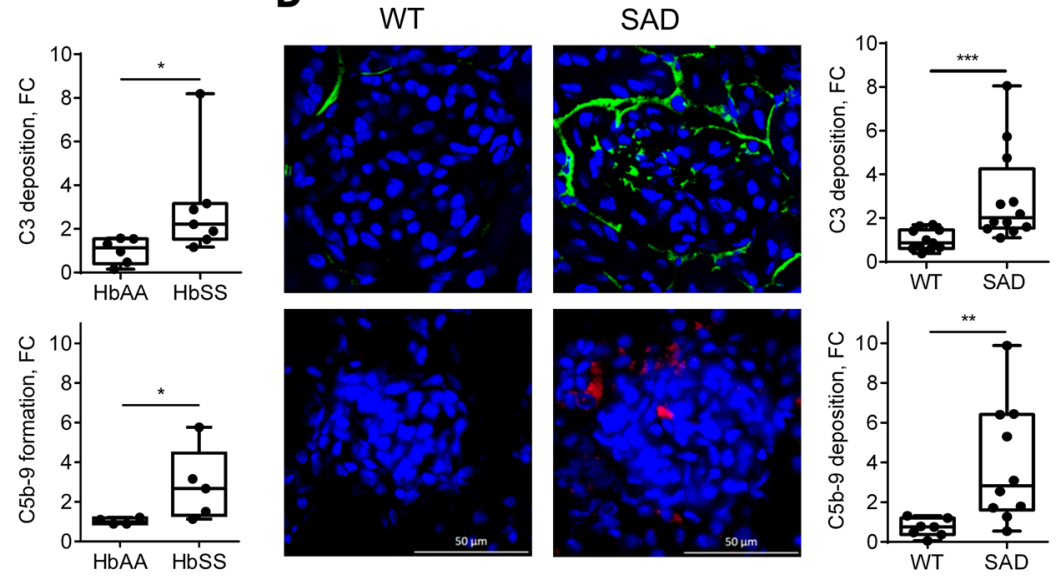

Figure 1. Patients suffering from SCD nephropathy and SCD mice have complement deposition within kidneys. (A) C3 fragment staining (false color green) and (B) C9 staining (brown) of kidney biopsies of patients with SCD nephropathy, performed by immunofluorescence of frozen tissue and immunohistochemistry of paraffin-embedded tissues, respectively. A normal protocol kidney allograft biopsy performed at 3 months was used as a negative control and a biopsy of a patient with acute humoral rejection was used as a positive control for the staining. (C) C3b/iC3b (false color green) and C5b-9 (false color red) staining of kidney sections of HbAA and HbSS mice. Quantification of the C3 and C5b-9 staining in HbSS ( $n \geq 7$ mice per group) and HbAA ( $n \geq 4$ mice per group) mouse kidney glomeruli. (D) C3b/iC3b (false color green) and C5b-9 (false color red) staining of kidney sections of SAD mice and WT littermates. Quantification of the C3 and C5b-9 staining in kidney glomeruli ( $n \geq 7$ mice per group). (E) Double staining of SAD and HbSS mouse kidney sections for C3b/iC3b (false color green) and endothelial marker CD31 (false color red). The merge image indicates colocalization in orange. One glomerulus was focused on. ${ }^{*} P<0.05 ;{ }^{*} P<0.005 ;{ }^{*} P<0.001$, Mann-Whitney test. Values are shown as box plots with median and minimum/maximum points. Scale bar: $50 \mu \mathrm{m}$. Original magnification, $\times 26$.

intravascular hemolysis (Supplemental Figure 2D). C3b/iC3b-positive staining was observed in glomeruli or around blood vessels and tubules within kidney sections of PHZ-treated mice, located in a similar fashion to SCD mice. The staining was specific, since it was completely absent from PBS- or PHZ-injected $\mathrm{C}^{-/-}$mice (Supplemental Figure 4). C3 deposits in glomeruli partially colocalized with EC marker vWF (Figure 2B). A similar pattern of complement activation was reproduced in the heart, 
A

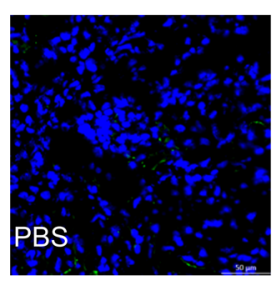

C3 fragments deposition

C

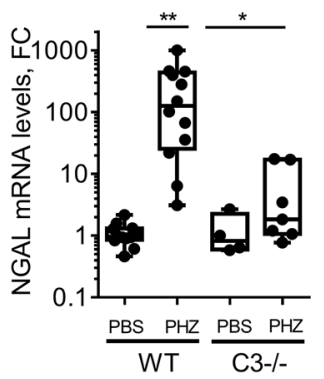

G

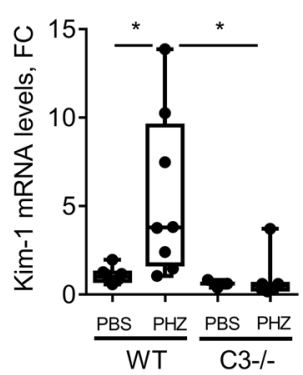

B

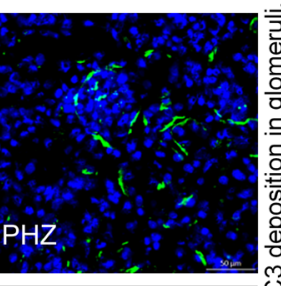

C3b/ic3b

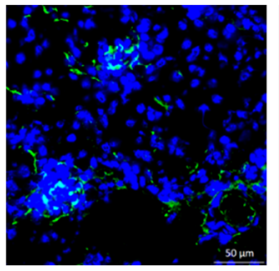

vWF

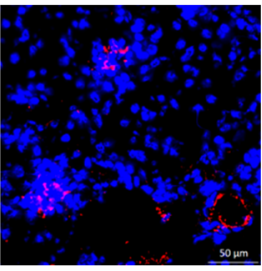

Merge

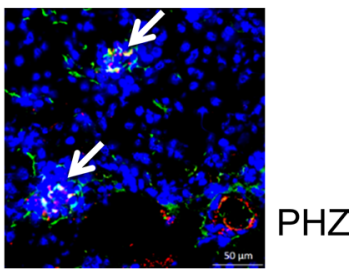

$\mathbf{F}$

E

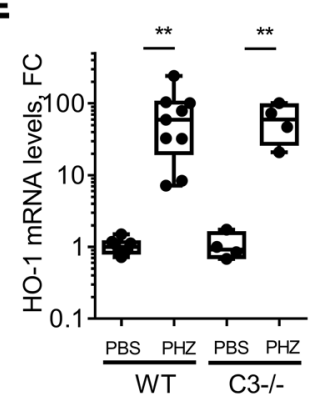

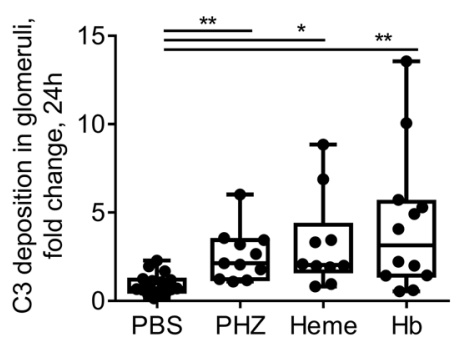

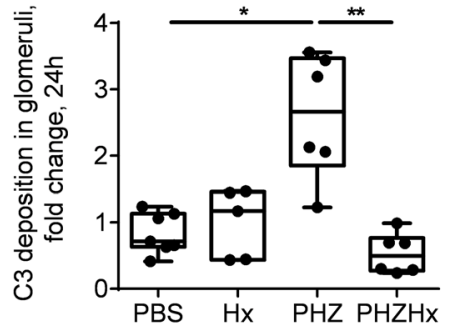

H

C3 fragments deposition
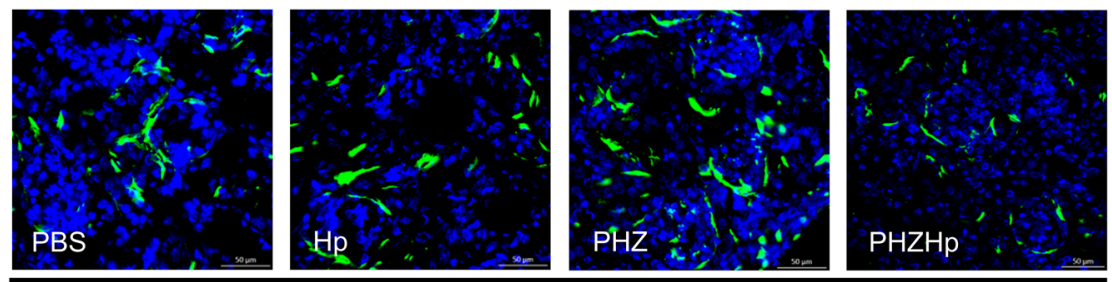

C3 fragments deposition

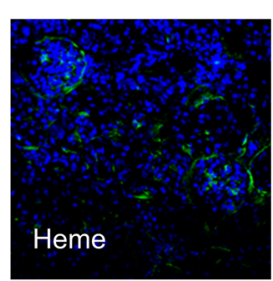

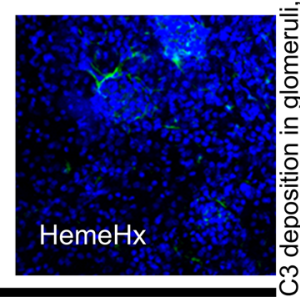

C3 fragments deposition

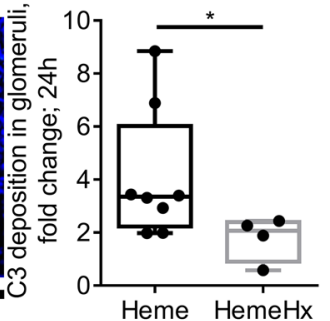

Heme HemeHx

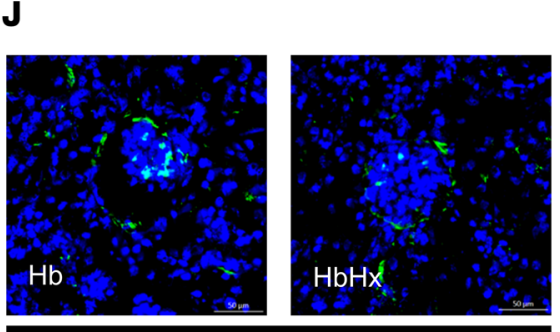

C3 fragments deposition

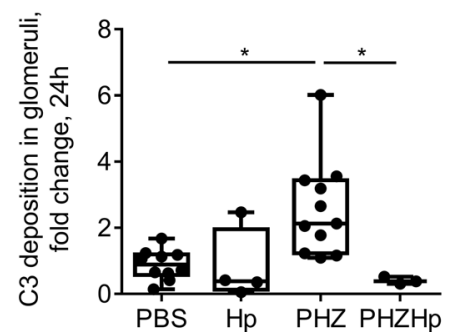

Figure 2. Hemolysis induced heme-dependent complement deposition in kidney in vivo. (A) C3b/iC3b (false green color) staining of frozen kidney sections of mice injected with PBS or PHZ and sacrificed at 6 hours. Quantification of C3 fragment staining in glomeruli ( $n \geq 5$ mice per group). (B) Double staining of $\mathrm{PHZ}$-injected mouse kidney sections for $\mathrm{C3b} / \mathrm{iC} 3 \mathrm{~b}$ (false color green) and endothelial marker VWF (false color red). The merge image indicates colocalization (white arrows) in orange. (C-E) Evaluation of the renal injury in WT and $\mathrm{C3}^{-1-}$ mice ( $n=$ between 4 and 12 mice per group) injected with PBS or PHZ by analyzing gene expression by RTqPCR. (C) NCAL. (D) Kim-1. (E) HO-1. (F) Comparison of the quantification of the C3 fragment staining in glomeruli of mice injected with PBS, PHZ, heme, or hemoglobin ( $\mathrm{Hb}$ ) ( $n \geq 10$ mice per group). (C) C3b/iC3b (false green color) staining and quantification in glomeruli of frozen kidney sections of mice injected with PBS or PHZ, with or without hemopexin $(\mathrm{Hx})$ at 24 hours after treatment. $(\mathrm{H}) \mathrm{C3b} / \mathrm{iC} 3 \mathrm{~b}$ (false green color) staining and quantification in glomeruli of frozen kidney sections of mice injected with PBS or PHZ, with or without haptoglobin (Hp) at 24 hours after treatment ( $n \geq 4$ mice per group). (I and J) C3b/iC3b staining (false color green) of kidney sections of mice injected with heme (I) or Hb (J), with or without Hx. Quantification of C3 staining in glomeruli ( $n \geq 4$ mice per group). ${ }^{*} P<0.05,{ }^{* *} P<0.005$, Mann-Whitney test (A, I, and J); Kruskal-Wallis with Dunn's test for multiple pairwise comparisons (F-H); 2 -way ANOVA with Tukey's test for multiple comparisons (C-E). Values are shown as box plots with median and minimum/maximum points. Scale bar: $50 \mu \mathrm{m}$. 
although with a lower apparent C3-staining density (Supplemental Figure 5). C5b-9 staining was negative (data not shown). Staining for $\mathrm{C} 3 \mathrm{~b} / \mathrm{iC} 3 \mathrm{~b}$ revealed that intact $\mathrm{RBCs}$ of PBS-, PHZ-, or hemeinjected mice remained largely negative (data not shown).

PHZ-treated mice presented with increased renal gene expression of NGAL and Kim-1, which are early and sensitive markers for renal injury (Figure 2, C and D). The expression of these genes was significantly decreased in $\mathrm{C}^{-/-}$mice, suggesting dependence on complement. In contrast, the gene expression of the cytoprotective gene $\mathrm{HO}-1$ was equally upregulated by $\mathrm{PHZ}$ in $\mathrm{WT}$ and $\mathrm{C}^{-/-}$mice (Figure $2 \mathrm{E}$ ).

To find out whether complement deposits in PHZ-treated mice are heme dependent or occur due to upstream products (such as $\mathrm{Hb}$ ), WT mice were injected with hemin and purified human $\mathrm{Hb}$, which also resulted in significant $\mathrm{C} 3$ deposits (Figure $2 \mathrm{~F}$ ). In addition, scavengers of heme (human plasma-derived $\mathrm{Hx}$, Figure 2G, confirmed as reaching the circulation, Supplemental Figure 2F) or Hb (human plasma-derived $\mathrm{Hp}$, Figure $2 \mathrm{H}$ ) were used. They both attenuated significantly and nearly completely the complement activation in kidneys of PHZ-injected mice. Interestingly, pretreatment with $\mathrm{Hx}$ prevented not only the complement deposits triggered by hemin (Figure 2I) but also by $\mathrm{Hb}$ (Figure 2J), showing that $\mathrm{Hb}$ acts via release of heme.

Heme triggers rapid EC surface complement deposition in vitro, which is counteracted by Hx and to a lesser extend by human serum albumin. The protective effect of $\mathrm{Hx}$ against complement deposition on ECs in vivo and in vitro could be related at least to two phenomena acting against targets in serum and on EC. We compared the efficacy of human serum albumin (HSA) and $\mathrm{Hx}$ in in vitro experiments using human umbilical vein endothelial cells (HUVECs). Two settings were used. First, heme was added with the serum-free culture medium, and after 30 minutes normal human serum (NHS) was added to the wells, while heme was still present (H+NHS condition). This condition evaluates the cumulative effect of the EC activation, which renders the cells susceptible to complement deposition, together with the direct activation of complement in serum. In the second setting, ECs were incubated with heme for 30 minutes, and after that the heme-containing supernatant (SN) was removed before addition of NHS (SN removed condition). This condition evaluates the complement activation due to modification of the EC surface only.

Hemin, but not $\mathrm{Hb}$, activated complement in fluid phase in sera and on the EC surface (Figure 3, A and $\mathrm{B}$ ). We observed that $5 \mu \mathrm{M} \mathrm{Hx}$ inhibited the effects of up to $50 \mu \mathrm{M}$ hemin on $\mathrm{C} 3$ and $\mathrm{C} 5 \mathrm{~b}-9$ deposition, while HSA had no effect at this dose and was overall 8- to 10-fold less efficient compared with Hx (Figure 3, C-F, and Supplemental Figure 6, A-E).

The inhibitory effect of different concentrations of $\mathrm{Hx}$ was compared with HSA, because of the high HSA concentrations in blood and its affinity, albeit low, for heme (Supplemental Figure 6, A-C). Exposure of ECs to heme with increased concentration of Hx prevented dose-dependent $\mathrm{C} 3$ fragment deposition on cell surface. Around $90 \%$ of the inhibition of the deposition caused by $50 \mu \mathrm{M}$ heme was obtained up to $10 \mu \mathrm{M} \mathrm{Hx}$ in both conditions - when SN was removed and when NHS was directly added to media in presence of heme (Supplemental Figure 6A). However, higher amounts of HSA were needed to inhibit C3 fragment deposition, since $40 \%$ of C3 fragment deposition remained detectable with $80 \mu \mathrm{M}$ HSA (Supplemental Figure 6A). Efficiency of HSA was similar when SN was removed or not, as measured by HSA titration (Supplemental Figure 6, B and $\mathrm{C})$. However, $\mathrm{Hx}$ was more efficient at low doses $(\leq 5 \mu \mathrm{M})$ when $\mathrm{SN}$ was removed. In order to compare the potential inhibitory effect of $5 \mu \mathrm{M}$ Hx with HSA, HUVECs were treated with $5 \mu \mathrm{M}$ HSA with increased concentration of heme. SN was removed before addition of NHS. C3 fragment deposition and C5b-9 formation were not controlled by the addition of $5 \mu \mathrm{M}$ HSA in the media (Supplemental Figure 6, D and E).

As previously observed by others $(4,20)$, our experiments in vitro showed that we did not observe a one-to-one neutralizing effect between $\mathrm{Hx}$ and hemin. This can be explained by the poor solubility of hemin, which tends to aggregate in aqueous solutions, decreasing its active concentration $(1,2)$.

$H x$ protects the ECs from acquiring a complement-activating phenotype. So far we have seen that the complement deposition resulting from $\mathrm{PHZ}$-induced hemolysis was heme dependent and responded to Hx. We therefore aimed to study this phenomenon in vitro in a rapid time frame (30 minutes) and to compare the effect of hemin and $\mathrm{Hb}$ as well as the scavenging by $\mathrm{Hx}$ and HSA. On the EC surface, hemin caused a dose-dependent decrease of $\mathrm{C} 3 \mathrm{~b}$ regulator MCP expression (up to 50\%), which was prevented by $\mathrm{Hx}$ (Figure 4, A and B). In contrast, equimolar HSA had no effect (Supplemental Figure 6F). Moreover, hemin induced rapid, Hx-sensitive expression of $\mathrm{P}$ selectin, a molecule known to recruit $\mathrm{C} 3 \mathrm{~b}$ on EC surfaces (Figure $4 \mathrm{C}$ ).

We detected a 2- to 2.5-fold increase in cells with high expression of anaphylatoxin receptors C3aR and C5aR, within 30 minutes, at $50 \mu \mathrm{M}$ hemin (Figure 4, D and F). This effect was dose dependent 
A
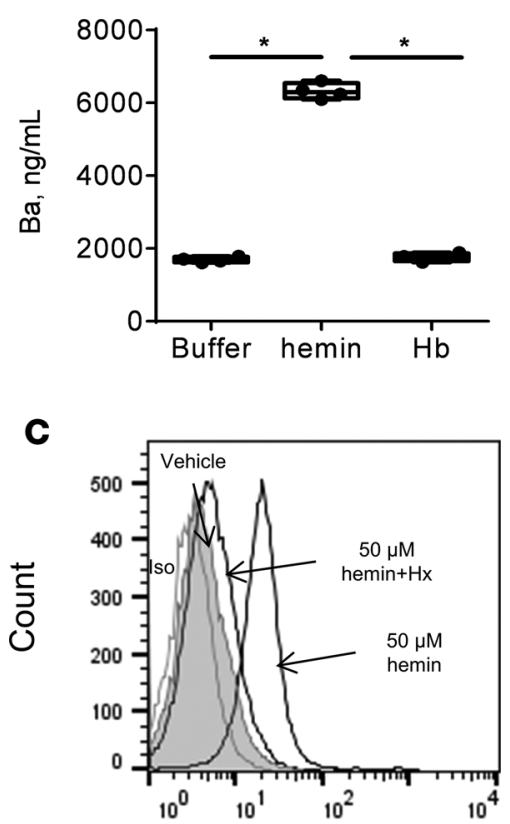

C3 deposition

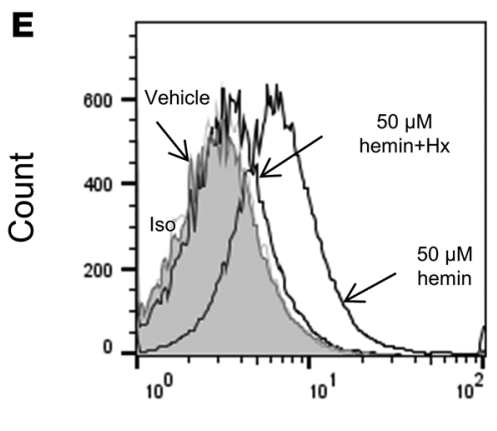

C5b-9 deposition
B

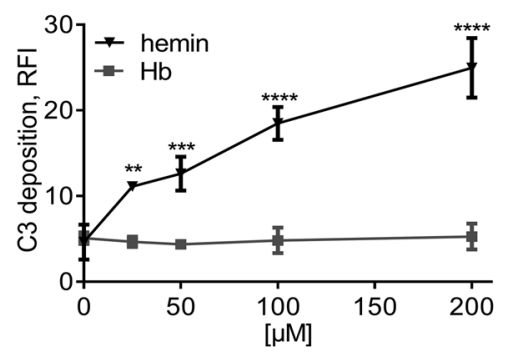

D

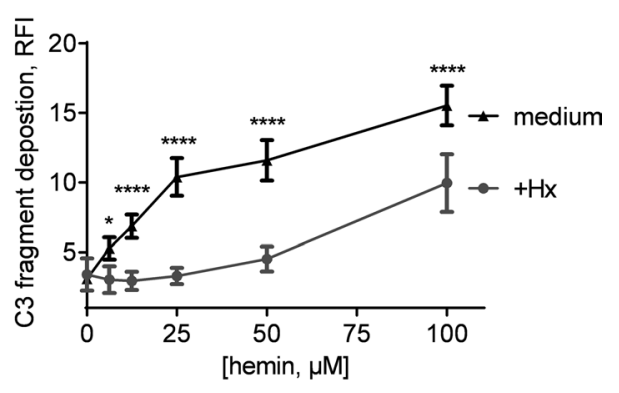

$\mathbf{F}$

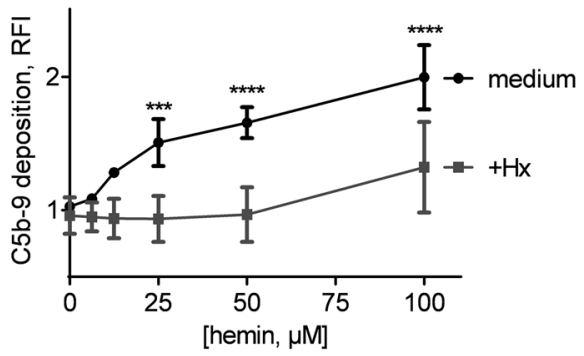

Figure 3. Heme and not $\mathrm{Hb}$ induced complement activation in serum and on EC surface, which was controlled by Hx. (A) NHS was incubated with $50 \mu \mathrm{M}$ hemin, $12.5 \mu \mathrm{M} \mathrm{Hb}$ (1 molecule of Hb contains 4 heme molecules) diluted in TBS. After a 30 -minute incubation time at $37^{\circ} \mathrm{C}$, the level of released $B$ a was measured by ELISA. ${ }^{*} P<0.005$, Kruskal-Wallis with Dunn's test for multiple pairwise comparisons. (B) HUVECs were treated with increasing concentrations of hemin or diluted in FCS-free culture medium (M199) for 30 minutes at $37^{\circ} \mathrm{C}$. After removing the supernatant, HUVECs were exposed to NHS (33\% final concentration diluted in the same medium) for 30 minutes at $37^{\circ} \mathrm{C}$. Cells were detached and stained for $\mathrm{C} 3$ deposition by flow cytometry. (C-F) HUVECs were treated with increased concentrations of hemin with or without $5 \mu \mathrm{M}$ of $\mathrm{Hx}$ for 30 minutes at $37^{\circ} \mathrm{C}$. After removing the supernatant, HUVECs were exposed to NHS (33\% final concentration diluted in M199 FCSfree) for 30 minutes at $37^{\circ} \mathrm{C}$. Cells were detached and stained for $\mathrm{C} 3$ deposition (C and $\mathbf{D}$ ) or $\mathbf{C 5}$ b-9 formation ( $\mathbf{E}$ and $\mathbf{F}$ ) by flow cytometry. (C and $\mathbf{E}$ ) Representative flow cytometry histograms. ( $\mathbf{D}$ and $\mathbf{F}$ ) Quantitative analyses. ${ }^{*} P<0.05,{ }^{* *} P$ $<0.005$, ${ }^{* *} P<0.001,{ }^{* * * *} P<0.0001$, 2-way ANOVA with Sidak's test for multiple comparisons. Values are shown as box plots with median and minimum/ maximum points.

(Figure 4, E and G). Hx at $5 \mu \mathrm{M}$ inhibited this increase, while equimolar HSA had no effect (Supplemental Figure 6, G and $\mathrm{H}$ ). The dotted lines in Figure 4, B, E, and G, represents the surface expression in absence of heme ( 0 heme in the titration curve).

$R B C$ membranes induce complement activation in a heme-dependent manner. RBC MVs may represent a significant and pathological form of heme carrier in plasma, particularly during intravascular hemolysis and more specifically in SCD (5). Such RBC MVs are present in our SCD mouse models (Supplemental Figure 1, A and B). We tested the potential capacity of human RBC membrane-derived MVs to activate complement, measured by Ba release, and the inhibitory effect of Hx. First, we compared the complement-activation capacity of RBC MVs generated in vitro using two different methods in order to trigger vesiculation: a $\mathrm{Ca}^{2+}$ ionophore or mechanical stimulation (shear stress). MVs prepared with the $\mathrm{Ca}^{2+}$ ionophore were selected for further studies due to the higher production yield (Supplemental Figure 7A).

Stimulating ECs with these MVs induced a dose-dependent increase in both $\mathrm{Ba}$ and sC5b-9 release (Supplemental Figure 7, B and C). SCD RBC MVs (which carried about twice the heme of control $\mathrm{RBC}$ MVs, as previously described, ref. 5) significantly increased $\mathrm{Ba}$ and $\mathrm{sC} 5 \mathrm{~b}-9$ release compared with healthy donor (HD) levels (Figure 5, A and B). There was a linear correlation of Ba with sC5b-9 release (Figure 5C). Hx significantly but partially decreased complement activation for SCD and HD RBC MVs (Figure 5, D and E, and Supplemental Figure 7D). The MVs expose phosphatidylserine, which is a potent (16) complement activator, explaining thus the fraction of heme-independent $\mathrm{Ba}$ and sC5b-9 
A

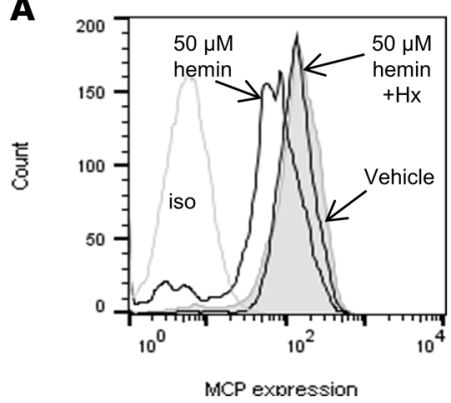

C

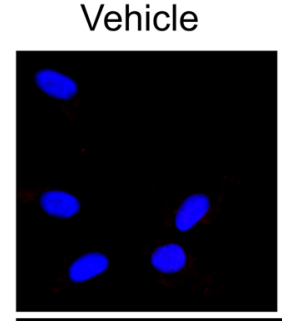

Heme

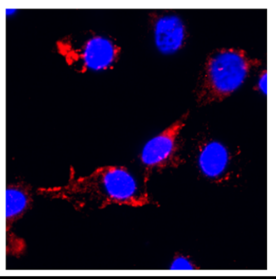

P-selectin expression
B

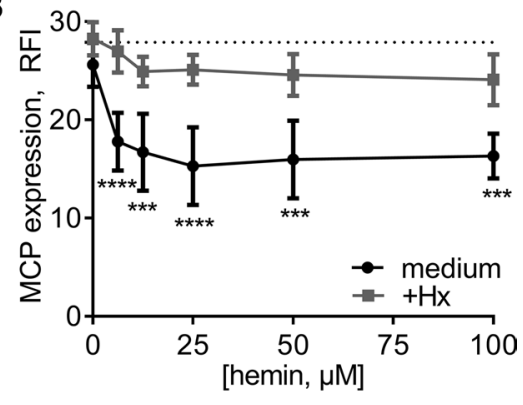

Figure 4. Heme induced complement-related stress markers on the EC surface, which can be controlled by Hx. HUVECs were treated with increasing concentrations of hemin with or without $5 \mu \mathrm{M} \mathrm{Hx}$ for 30 minutes at $37^{\circ} \mathrm{C}$. Cells were detached and stained for MCP (A and B), C3aR (D and E), or C5aR (F and $\mathbf{G}$ ) expression by flow cytometry. ${ }^{*} P<0.05$, ${ }^{*} P<0.005$, ${ }^{*}{ }^{*} P<0.001$, ${ }^{* *}{ }^{*} P$ $<0.0001,2$-way ANOVA with Sidak's test for multiple comparisons. (A, D, and F) Histograms from flow cytometry. (B, E, and G) Quantitative analyses. (C) HUVECs were treated with $50 \mu \mathrm{M}$ heme with or without $5 \mu \mathrm{M} \mathrm{Hx}$. Expression of $P$ selectin (false red color) was stained and studied by immunofluorescence. Nuclei are stained with DAPI (blue). Original magnification, $\times 63$.

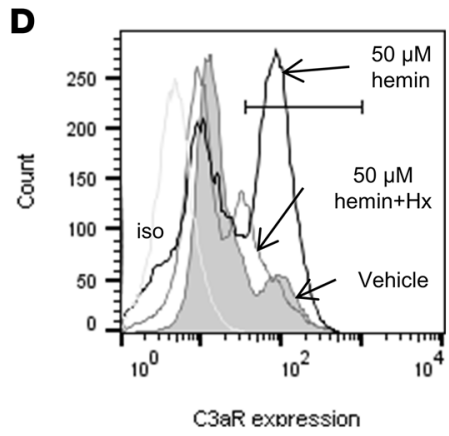

E
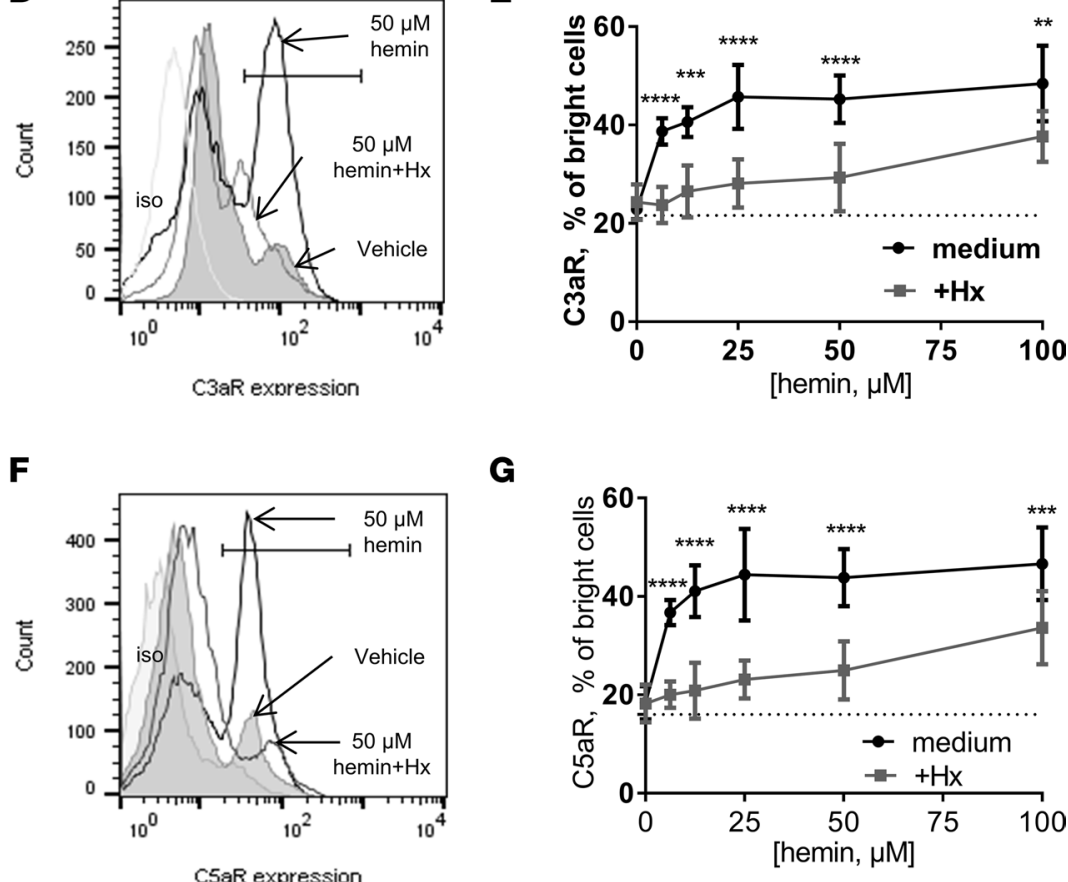

G
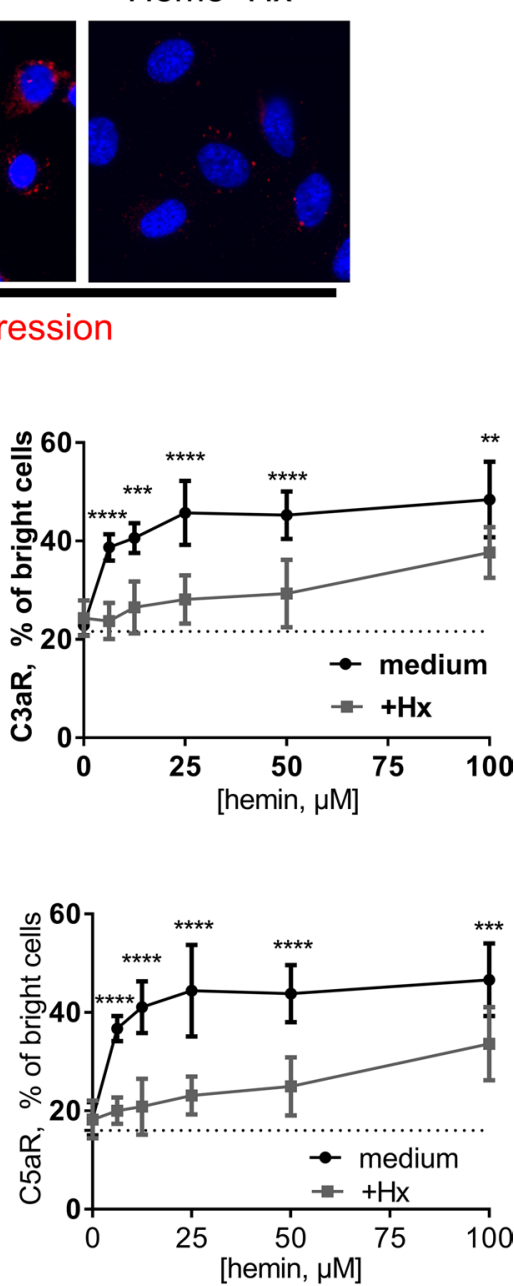
A

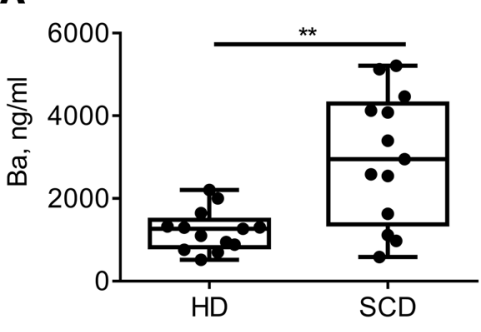

D

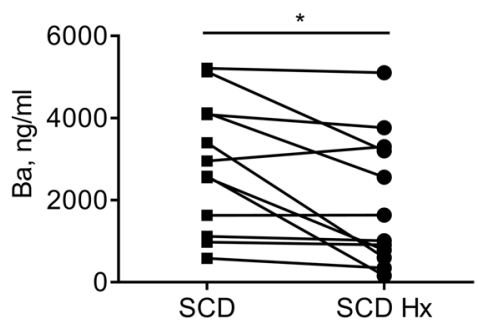

$\mathbf{F}$

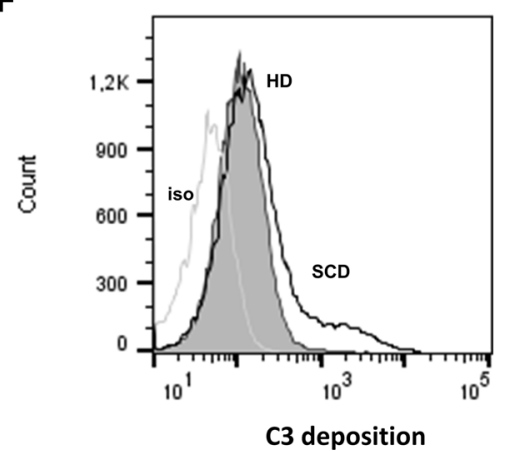

B

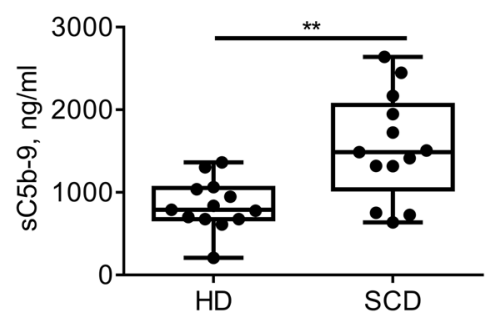

E

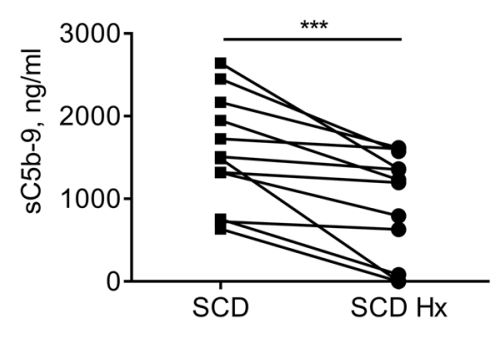

G

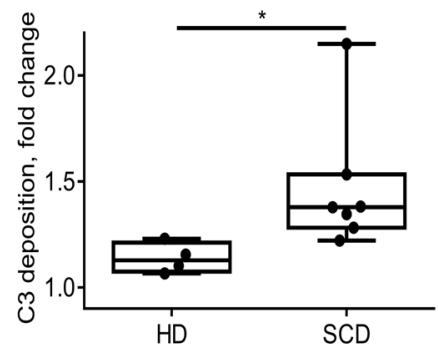

E
C

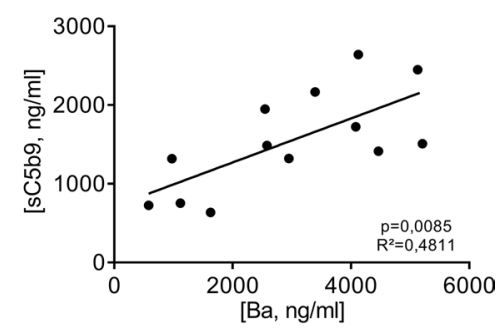

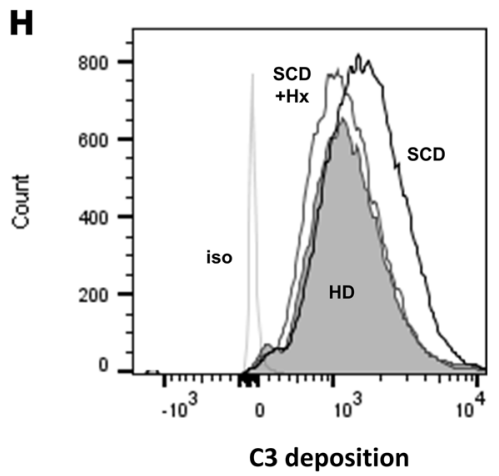

Figure 5. Erythrocyte MPs from SCD patients activated complement in a heme-dependent, Hx-sensitive manner. (A and B) NHS was incubated with $1,000 \mathrm{MV} / \mu \mathrm{l}$ RBC MVs from HDs $(n=12)$ or SCD patients $(n=12)$ diluted in TBS. After 30 minutes at $37^{\circ} \mathrm{C}$, the levels of released Ba $(\mathbf{A})$ and sC5b-9 $(\mathbf{B})$ were measured by ELISA. ${ }^{* *} P<0.005$, Mann-Whitney test. (C) Linear correlation between measured Ba and sC5b-9 levels with RBC MPs from SCD ( $\left.n=12\right)$ in tested samples. $\mathrm{R}^{2}=0.4811 ; P=0.0085$. ( $\mathbf{D}$ and $\left.\mathbf{E}\right)$ NHS was incubated with $1,000 \mathrm{MV} / \mu \mathrm{MV}$ s from SCD patients $(n=12)$ diluted in TBS with or without $25 \mu \mathrm{M} \mathrm{Hx}$. After 30 minutes at $37^{\circ} \mathrm{C}$, the levels of released $\mathrm{Ba}(\mathbf{D})$ and $\mathrm{sC} 5 \mathrm{~b}-9$ (E) were measured by ELISA. ${ }^{*} P<0.05$, ${ }^{* * *} P<0.001$, Wilcoxon test after a Shapiro-Wilk test for normality. (F and G) HUVECs were treated with 1,000 MV/ $\mu$ RBC MPs from HDs $(n=4)$ or SCD patient $(n=7)$ diluted in M199 medium for 30 minutes at $37^{\circ} \mathrm{C}$. Without removing the supernatant, HUVECs were exposed to NHS (final concentration at $33 \%$ diluted in M199 FCS-free) for 30 minutes at $37^{\circ} \mathrm{C}$. Cells were detached and stained for $C 3$ deposition by flow cytometry. (F) Flow cytometry histograms. (C) Quantitative analyses. ${ }^{*} P<0.05$, Mann-Whitney test. (H) HUVECs were treated with 1,000 MV/ $\mu$ I RBC MPs from 1 SCD patient with or without $5 \mu M$ Hx. As above, NHS was added and cells were stained for $\mathrm{C} 3$ deposition by flow cytometry. Values are shown as box plots with median and minimum/maximum points.

a concentration of $1,000 \mathrm{SCD}$ MVs/ $\mu 1$ had comparable effects to $6 \mu \mathrm{M}$ free hemin. The concentration of heme previously reported in a similar preparation of RBC MVs approximated $5 \mu \mathrm{M}(5)$.

Hemolysis and heme activate complement by the alternative pathway. We confirmed here that hemin activates complement by the alternative pathway (AP) in NHS $(11,12)$ (Supplemental Figure 8C), since the sC5b-9 release in presence of $\mathrm{Ca}^{2+}$ chelator EGTA and $\mathrm{Mg}^{2+}$ (allowing activation of the AP only) was identical to that in the presence of $\mathrm{Ca}^{2+}$ and $\mathrm{Mg}^{2+}$ in the buffer (allowing activation of all pathways). Similar results were obtained for HD MVs and MLVs (Supplemental Figure 8, A, B, and D). Moreover, $\mathrm{Ba}$, which is a specific marker of the AP, was released after addition of RBC MVs in sera and is sensitive to Hx (Figure 5, A and D).

To evaluate the contribution of the AP in vivo, we induced hemolysis in $\mathrm{C}^{-\mathrm{q}^{--}}$mice, which are unable to activate the classical pathway. C3 deposits in kidneys were still present despite C1q deficiency (Supplemental Figure 8E). 


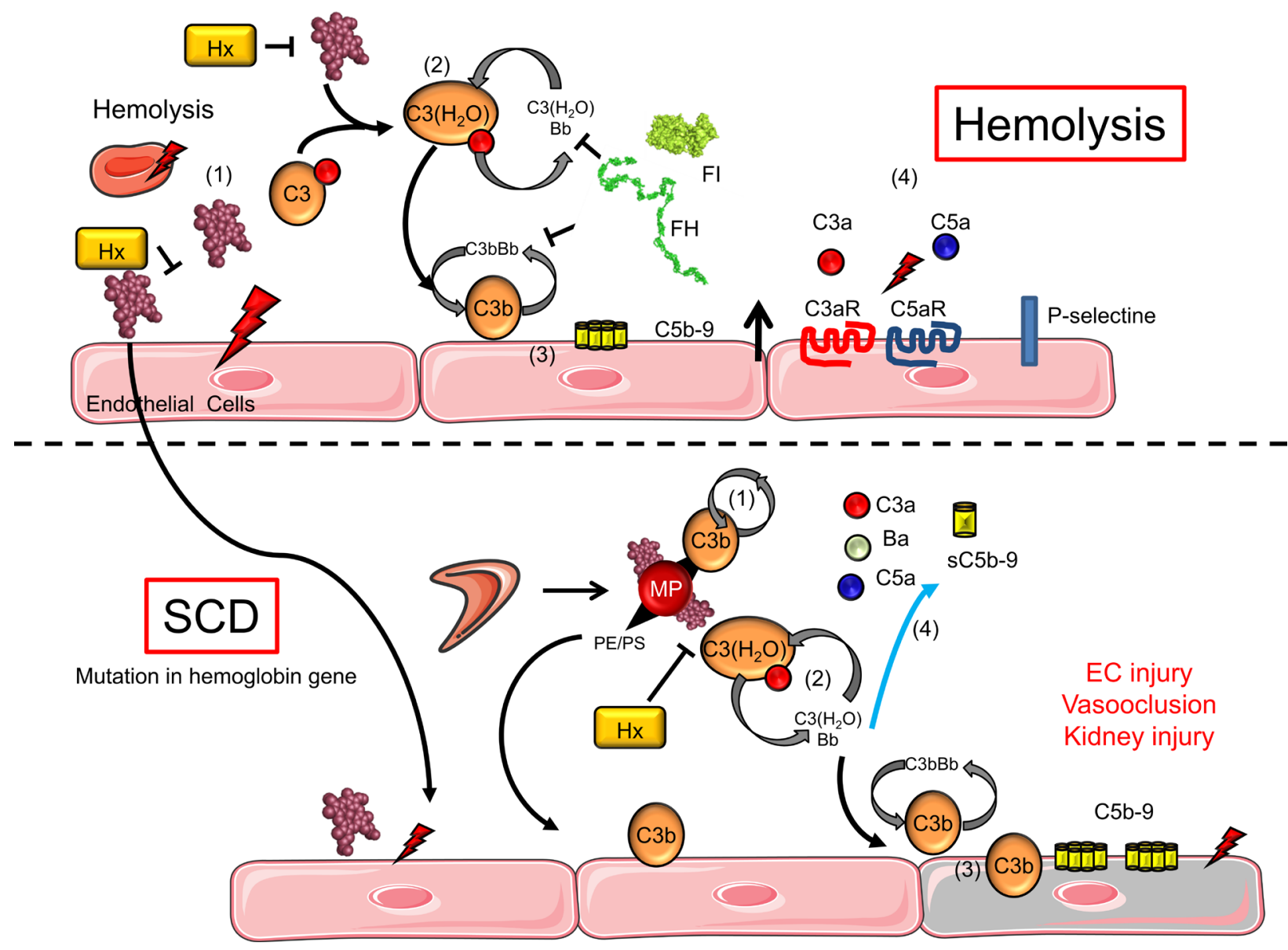

Figure 6. Schematic view of different nonexclusive mechanisms leading to complement activation during hemolysis. Extracellular heme can serve as an enhancer of the pathological process in SCD and other hemolytic diseases. (i) Heme activates EC by its proinflammatory and prooxidant effects. (ii) Heme induces hydrolysis of $\mathrm{C} 3$ into $\mathrm{C} 3\left(\mathrm{H}_{2} \mathrm{O}\right)$ and provokes direct complement activation in the serum. (iii) $\mathrm{C} 3 \mathrm{~b}$ generated by the fluid-phase $\mathrm{C} 3$ convertase will bind to the EC membrane and will form new AP C3 convertases that can locally autoamplify and generate C3b and C5b-9 deposition. (iv) Released C3a and C5a can bind to C3aR and C5aR to mediate proinflammatory processes. In the context of SCD, sickle RBCs will generate more heme-positive MVs. They carry heme, which will activate complement in serum and also on the EC surface. We hypothesize that this complement activation will contribute to the vascular injury and tissue damage in SCD. Hx was able to block, partially or completely, the hemolysis-induced complement activation in vitro and in vivo.

\section{Discussion}

The results presented here helped decipher the mechanisms of complement activation triggered by intravascular hemolysis. Complement activation in kidneys is a key factor for local inflammation and vascular and tissue damage in a large spectrum of renal diseases (21). C3 fragment deposition within tissues represents a durable signal of tissue inflammation as well as a sensitive and robust indicator of disease activity (22). On the other hand, intravascular hemolysis plays a key role in the pathogenesis of renal dysfunction in SCD (23).

We observed significant C3 deposits in human SCD nephropathy biopsies and in kidneys of two mouse models of SCD. Our study of plasma heme and $\mathrm{Hb}$ levels confirmed significant levels of intravascular hemolysis and cell-free heme of both models. A similar pattern of C3 fragment deposition was observed in experimental intravascular hemolysis ( $\mathrm{PHZ}$ induced). Moreover, we report glomerular C5b-9 deposits in human and mouse SCD kidneys for the first time to our knowledge. We concluded that complement activation is associated with intravascular hemolysis and, more specifically, the increase in cell-free heme in plasma. In addition, this pathway progresses up to its terminal phase, with membrane attack complex formation in SCD.

We investigated the molecular connection between intravascular hemolysis and complement activation. We found that injection of $\mathrm{Hb}$ or heme both induced complement deposition, and these deposits were attenuated by pretreatment with $\mathrm{Hp}$ and $\mathrm{Hx}$. In our experiments, Hx prevented complement activation mediated by $\mathrm{Hb}$. This indicated that $\mathrm{Hb}$ operates indirectly via liberation of heme. In fact, the induction of hemolysis rapidly activated complement and endogenous $\mathrm{Hx}$ levels dropped at the same time, suggesting a direct link 
between the increase in cell-free heme and Hx consumption. From this group of data, we concluded that intravascular hemolysis triggered complement activation, in a process mediated by the release of $\mathrm{Hb}$ by RBCs, which was dependent on the activation of cell- and globin-free heme, likely released during spontaneous $\mathrm{Hb}$ oxidation in plasma. Deposits persisted despite the Hx normalization and HO-1 expression at 24 hours, due to the covalent attachment of $\mathrm{C} 3 \mathrm{~b} / \mathrm{iC} 3 \mathrm{~b}$ to the cell surface. Existing literature includes proteomic analyses of kidneys from old blood-transfused hemolytic mice and revealed increased presence of complement proteins of the AP (Factors C3, B, and D); those were attenuated by Hp administration (24). Here, complement activation in response to intravascular hemolysis progressed through the AP, despite suspected effects of heme on immunoglobulins, or the potential activation of the classical pathway (2, 20, 25-27). This corroborates previous clinical reports of increased AP activation marker Bb and sC5b-9 complexes in SCD patient sera (13-15).

We simulated the secondary mediators released by RBCs during intravascular hemolysis. We studied complement activation in vitro in response to heme, $\mathrm{Hb}$, and $\mathrm{RBC}$-derived MVs. Once released in the circulation, heme is likely to bind promptly to one of its multiple proteic or lipidic partners, due to its highly hydrophobic nature. RBC-derived membrane MVs represent a significant form of heme carrier in plasma during intravascular hemolysis (5), and their levels increase about 3-fold during VOC in SCD (28, 29). RBC-derived MVs contribute to chronic inflammation in SCD as well as transfusion-related complications $(27,30)$. RBC-derived MVs are sufficient to trigger VOCs in SAD mouse kidneys, in a heme-dependent and Hx-inhibited process $(5,7)$. Here, RBC-derived MVs from SCD patients activated the alternative and terminal complement pathways. Artificial heme-loaded MLVs also triggered complement activation substantially, compared with MLVs alone, confirming that the MV pathway leading to complement activation is partially mediated by heme. The established presence of RBC-derived MVs in SCD patients, SAD and HbSS mice, as well as in PHZ-injected WT mice, combined with our observation of a direct, heme-dependent complement activation by human RBC MVs, suggests that RBC-derived MVs are a pathologically relevant form of heme carrier involved in complement activation.

In cultured ECs, C3 and C5b-9 deposition occurred upon brief exposure to heme but not $\mathrm{Hb}$ and could be prevented by the use of $\mathrm{Hx}$. These deposits could be explained by a rapid cell surface expression of $\mathrm{P}$ selectin. $\mathrm{P}$ selectin is known to recruit $\mathrm{C} 3 \mathrm{~b}(31,32)$ and represents an interesting therapeutic target as a mediator of blood cell adhesion to vessel walls and VOC in $\operatorname{SCD}(33,34)$. When exposed to heme, ECs partly lost C3b-regulating MCP and increased their expression of C3aR and C5aR. This supports the C3aR upregulation reported in transfusion-induced hemolysis (35). Hence, hemolysis, and heme in particular, unlike $\mathrm{Hb}$ alone, may enhance the responsiveness of ECs to complement-mediated inflammation. High levels of soluble $\mathrm{C} 3 \mathrm{a}$ and $\mathrm{C} 5 \mathrm{a}$ can then bind to $\mathrm{C} 3 \mathrm{aR}$ and $\mathrm{C} 5 \mathrm{aR}$ to mediate pro-inflammatory processes. We have previously shown that heme induces hydrolysis of $\mathrm{C} 3$ into $\mathrm{C} 3\left(\mathrm{H}_{2} \mathrm{O}\right)$ and provokes direct complement activation in sera $(2,9-12)$. C3b generated by the fluid-phase $\mathrm{C} 3$ convertase will bind to EC membranes and form new $\mathrm{C} 3$ convertases of the AP, which can autoamplify locally and increase C $3 \mathrm{~b}$ and C5b-9 deposition.

Together, our data established that intravascular hemolysis induces the activation of the complement $A P$ via $\mathrm{RBC}$ degradation products, and EC sensitization to complement, in transgenic mouse models with SCD and in SCD patient biopsies (Figure 6).

Our data points at potential avenues to target inflammatory processes during intravascular hemolysis. One such possibility is indirect complement inhibition by heme-scavenging approaches. The experimental injection of $\mathrm{Hx}$ prior to $\mathrm{PHZ}, \mathrm{Hb}$, or heme administration clearly attenuated complement deposition, demonstrating the causal role of cell-free, globin-free heme. In vitro experiments showed that $\mathrm{Hx}$, and HSA at much higher concentrations, protected ECs from heme-mediated stress and subsequent complement activation in vitro. HSA is present at higher levels in plasma compared with $\mathrm{Hx}$ and can boost heme scavenging and protection against complement activation. Nevertheless, $\mathrm{Hx}$, but not HSA, was effective at preventing tissue injury and inflammation in mouse models of hemolysis (5, 36-39). Previous reports have also shown that hepatic overexpression of $\mathrm{Hx}$ inhibits inflammation and vascular stasis in murine SCD (40), while $\mathrm{Hx}^{-/-}$mice develop a severe renal damage after experimental hemolysis. This points to $\mathrm{Hx}$ as a guardian of kidney function during intravascular hemolysis (41). Hx could be also useful in cases of cardiac dysfunction subsequent to hemolysis. Indeed, in a model of repetitive injections of PHZ, oxidative stress in the heart was counteracted with preventive injections of $\mathrm{Hx}$ (39). The recent discovery of a specific antibody against heme, $2 \mathrm{H} 10$, will provide further information about the contribution of heme to the pathological consequences of hemolytic diseases (42). Benefits of Hx reflect deleterious effects of heme. Therapeutic Hx administration to alleviate heme-induced inflammation is under intense scrutiny, particularly in SCD (5, 36-39). However, Hx 
administration remained insufficient to prevent inflammation and renal injury in an old blood transfusion model of hemolysis $(5,36-39)$ as well as in our pharmacological model of hemolysis (43). Acute intravascular hemolysis may generate other RBC-derived products ( $\mathrm{Hb}, \mathrm{MVs}$, etc.) in quantities sufficient to sustain tissue injury independently from heme. Indeed, hemolysis and MV formation during blood storage and in patients receiving a transfusion of aged RBCs contribute to decreased NO bioavailability and vascular dysfunction in a $\mathrm{Hb}$-dependent manner (44). Conversely, $\mathrm{Hp}$ administration decreased renal injury significantly in the transfusion model $(5,36-39)$.

Taken together, our results and the data from the literature point toward a complex role of RBC degradation products in renal damage. It is well established that SCD nephropathy is a multifactorial process, related to NO scavenging by Hb, ROS generation, and heme-mediated injury (44-48). Here, we add heme-mediated complement activation as additional mechanism, potentially contributing to SCD kidney injury. Evidence for a pathogenic role of complement activation in hemolysis-induced renal manifestations comes from the observed attenuation of the expression of NGAL and Kim-1 in complement C3-deficient mice. Moreover, targeting C5 with a blocking antibody was shown to inhibit VOCs in transgenic mice with SCD (49), while inhibiting C3 with compstatin CP40 has been investigated in vitro in a model of malaria $(50,51)$. However, CP40 is still unavailable for clinical use. Complement inhibition downstream of C3 is now possible in the clinic, using the monoclonal, C5-blocking antibody eculizumab, which abrogates terminal pathway activation. Cases supporting the efficacy of eculizumab against delayed transfusion reaction and hemolysis in SCD are emerging (52), showing the importance of complement in the disease. Although we detected complement deposits in SCD nephropathy biopsies and mouse models of SCD, our results do not provide direct proof that complement is implicated in the renal injury. Nevertheless, our data urge for further studies in order to determine how much complement activation contributes to the pathological processes in chronic hemolytic diseases, including SCD nephropathy.

In summary, our study demonstrates alternative and terminal complement pathway activation during intravascular hemolysis and in SCD. It also suggests that heme-scavenging strategies, such as Hx supplementation, could indirectly help target complement activation (Figure 6).

\section{Methods}

\section{Reagents}

The $\mathrm{Fe}^{3+}$ form of heme (hemin [ferriprotoporphyrin IX], designated as heme (Frontier Scientific Inc. or MilliporeSigma) was dissolved to $20 \mathrm{mM}$ in $50 \mathrm{mM} \mathrm{NaOH}$ and $145 \mathrm{mM} \mathrm{NaCl}$ and further diluted in an appropriate vehicle just before use.

Stock solution of $25 \mathrm{mg} / \mathrm{ml}$ PHZ (MilliporeSigma) was prepared in PBS immediately before use. Plasma-purified Hx, Hp, and HSA were provided by CSL Behring. Complement ELISA kits MicroView Ba and sC5b-9 were from Quidel. MVs were obtained from SCD patients or HD RBCs after vesiculation with calcium ionophore A23187 or mechanical vesiculation as previously described (7).

\section{Patients}

Patients. Kidney biopsies and corresponding clinical data from 13 SCD patients were retrieved from the archives of the Necker-Enfants Malades Hospital, Paris, France $(n=10)$, and the Pathology Institute of the CHRU Lille, Lille, France $(n=3)$, to constitute a cohort of SCD nephropathy (Table 1) A normal protocol kidney allograft biopsy, performed at 3 months, was used as a negative control. A biopsy of a patient with acute humoral rejection and another from a patient with lupus nephritis were used as positive controls for the staining.

Classification of SCD patients. Kidney biopsy of 13 SCD patients is detailed in Table 1 . In 6 cases, kidney biopsies were performed because of chronic renal failure, and in the remaining 7 cases, kidney biopsies were performed because of proteinuria. The grading scale used was as follows: -, no lesion; +, rare or few lesions; ++ , moderate lesions; and +++ , severe lesions.

Staining for complement in patient kidney biopsies. Routine staining protocol for C3 (polyclonal rabbit antiC3c Human Complement/FITC, Dako, F0201) was applied for the patients with missing archived images. For the 3 patients from CHRU Lille, C9 staining was also available (rabbit anti-human C9, Abcam, ab71330) using the Ventana XT autostainer (Ventana Medical Systems, BenchMark XT). Whole slides were scanned and analyzed by an Axio Scan (Zeiss) or Nanozoomer (Hamamatsu). 


\section{Animal experimentation}

Mouse treatment. C57BL/ 6 mice were from Charles River Laboratories, and C1q ${ }^{-/-}$and C3 ${ }^{-/-}$mice (C57BL/6 background) were from an in-house colony, which was a gift from Marina Botto (Imperial College London, London, United Kingdom) and Sebastien Lacroix-Desmazes (Cordeliers Research Center, Paris, France). Eight-week-old female C57BL/6 WT mice were injected i.p. with $200 \mu 1$ PBS (Gibco) or PHZ (900 $\mu \mathrm{mol} /$ $\mathrm{kg}$, corresponding to $0.125 \mathrm{mg} / \mathrm{g}$ body weight), and the mice were sacrificed at 6 or 24 hours. WT C1q ${ }^{-1-}$ and $\mathrm{C}^{-/-}$mice were injected with the same dose of $\mathrm{PHZ}$ and sacrificed at 24 hours. Alternatively, PBS, 40 $\mu \mathrm{mol} / \mathrm{kg}$ freshly prepared heme (corresponding to $26 \mu \mathrm{g} / \mathrm{g}$ body weight, ref. 6 ), or $12.5 \mu \mathrm{mol} / \mathrm{kg}$ freshly prepared human $\mathrm{Hb}$ (corresponding to $800 \mu \mathrm{g} / \mathrm{g}$ body weight) was injected, and the mice were sacrificed at 24 hours. To test the efficacy of $\mathrm{Hx}$, mice were pretreated with i.p. injection of $0.491 \mu \mathrm{mol} / \mathrm{kg}$ human plasma-derived Hx (corresponding to $28 \mu \mathrm{g} / \mathrm{g}$ body weight, ref. 36) diluted in PBS 1 hour before i.p. injection of PBS or freshly prepared heme, $\mathrm{Hb}$, or PHZ, as above. The efficacy of Hp was tested by pretreatment of the mice with $1 \mu \mathrm{mol} / \mathrm{kg}$ human plasma-derived $\mathrm{Hp}$ (corresponding to $250 \mu \mathrm{g} / \mathrm{g}$ body weight) diluted in PBS 1 hour before i.p. injection of PBS or PHZ. Concentrations and route of administration were chosen as described previously $(6,36,39)$. Blood was collected from the submandibular vein 3 days before the first injection (baseline), at 6 hours, and before endpoint (day 1).

$S A D$ mice. SAD mice carry a human $\mathrm{Hb} \beta$ chain transgene with 3 mutations (bS b6Val, bS-Antilles, b23Ile, and D-Punjab b121Glu) on a C57BL/6J background; they are a well-characterized model of SCD and SCD-associated renal injury (17). Frozen kidneys from 6-month-old SAD mice were used for this study.

HbSS mice (Townes). HbSS mice express human $\alpha$-globin gene on a C57BL/ 6 background; they are a well-described model of SCD (53). Frozen kidneys from 3-month-old HbAA and HbSS mice were used for this study.

Immunofluorescence of mouse tissue. Five- $\mu$ m-thick frozen sections of kidneys, hearts, and spleens were cut with Cryostat Leica AS-LMD and fixed in acetone on ice for 10 minutes. Complement deposition was studied using rat anti-mouse C3 fragments (C3bi/C3b/C3c) IgG1 (Hycult Biotech, HM1065), visualized with goat anti-rat-AF488 (Invitrogen, A-11006) and rabbit anti-mouse C5b-9 (Abcam, ab55811), and visualized with goat anti-rabbit IgG-AF488 (Invitrogen, A-11008). Double staining for C3 deposition and EC marker CD31 was performed using rabbit anti-mouse CD31 (Abcam, ab28364), revealed by anti-rabbit AF555 (Invitrogen, A-21429). Stained slides were scanned by Slide Scanner Axio Scan (Zeiss) or analyzed by confocal microscopy (Confocal LSM710, Zeiss). Quantification of the staining was performed using Visiopharm software (Visiopharm A/S) by measuring the fluorescence intensity of the staining within the cortex reported on the total cortical surface.

Immunohistochemistry of mouse tissues. Five- $\mu$ m-thick frozen sections of kidneys and hearts were cut with Cryostat Leica AS-LMD and fixed in acetone on ice for 10 minutes. Complement deposition was studied using rat anti-mouse C3 fragments (C3bi/C3b/C3c) IgG1 (Hycult Biotech, HM1065), followed by a goat anti-rat polyclonal IgG-HRP (R\&D Systems). HO-1 expression was studied using rabbit anti-mouse HO-1 (Abcam, Ab13243), followed by a polymer anti-rabbit (DAKO, K4003). Both stainings were revealed with DAB solution. Slides were scanned by Nanozoomer (Hamamatsu). Hematoxylin and eosin and Perls' coloration were performed by routine procedures using sections of paraffin-embedded kidneys. Coloration of slides was scanned by Slide Scanner Axio Scan (Zeiss).

$m R N A$ level analyses. Frozen kidney sections were recovered in RLT buffer (Qiagen) $+1 \% \beta$-mercaptoethanol (Gibco) and used for mRNA extraction using Qiagen RNeasy miniKit. The quality and quantity of mRNA were evaluated with the Agilent 2100 bioanalyzer using the Agilent TNA 6000 NanoKit, followed by retrotranscription to cDNA. Gene markers of early kidney injury, NGAL, Kim-1, and the cytoprotective gene HO-1, relevant for hemolysis were analyzed by RTqPCR (ThermoFisher).

Quantification of cell-free heme, $\mathrm{Hb}$, and MVs in plasma. An approximation of extracellular heme levels in blood was obtained by measuring absorbance at $398 \mathrm{~nm}$ in mouse plasma, i.e., at the peak of Soret band absorbance. Straightforward absorbance in plasma can be somewhat affected by turbidity, but it is assumed that strong and sudden increases obtained in transgenic mice with SCD-like phenotypes, such as HbSS and SAD mice, will be mostly related to heme, as previously reported $(5,7)$. This technique will detect all forms of heme, whether it is retained within $\mathrm{Hb}$ or bound to other plasma partners and will reflect intravascular hemolysis accurately, which is further confirmed here with the PHZ-induced model. To confirm the occurrence of intravascular hemolysis, we assessed the levels of $\mathrm{Hb}$ in plasma using a completely different principle and a mouse Hemoglobin ELISA Kit (Abcam, ab157715), following the manufacturer's protocol. 
In addition to heme and $\mathrm{Hb}$, we evaluated the circulating levels of membrane-derived extracellular MVs in the plasma of our hemolysis models, with our well-established method (5, 7). Briefly, MVs present in plasma were labeled with fluorescent recombinant annexin A5 (labeled with FITC, Merck), analyzed by fluorescence-assisted cell sorting using an LSR II flow cytometer (BD Biosciences), and compared with size-calibrated microbeads (MegamixPlus Biocytex) and fluorospheres (Flow-Count, Beckman Coulter) to calibrate volume. In some experiments, plasma were depleted from MVs by ultracentrifugation $(20,500 \mathrm{~g}$ for 4 hours; Eppendorf 5804R). Pelleted MVs were discarded, leaving behind any material not bound to MVs.

Flow cytometry assessment of $C 3 b / i C 3 b$ on mouse RBCs. Blood from PBS-, PHZ-, or heme-injected mice was recovered 24 hours after injection and RBCs were purified. These cells were stained for C3 fragment deposition using rat anti-mouse C3bi/C3b/C3c IgG1 (Hycult Biotech, HM1065) and visualized with goat anti-rat AF488 (Invitrogen, A-11006). Alternatively, RBCs from naive mice were purified and incubated at $1 \times 10^{6}$ cells/tube for 30 minutes with $50 \mu \mathrm{M}$ heme, followed by addition of normal mouse serum for 30 minutes. Cells were washed and probed for C3 fragment deposits by flow cytometry (BD LSR II) and further analyzed by FlowJo X.

Hx quantification. Ten $\mu 1$ of plasma sample was placed into a clean Eppendorf tube followed by the addition of $75 \mu \mathrm{M} \mathrm{MeOH}$ to precipitate the protein. The methanol was removed after centrifugation. The pellet was air dried and afterward resuspended in $50 \mathrm{mM} \mathrm{NH}_{4} \mathrm{HCO}_{3} / 0.16 \%$ ProteaseMAX (Promega) containing heavy-isoptope labeled peptides, which are specific for human $\mathrm{Hx}$ or endogenous $\mathrm{Hx}$ and used as internal standards. Samples were incubated at $56^{\circ} \mathrm{C} / 550 \mathrm{rpm}$ for 45 minutes, reduced by adding $0.5 \mathrm{M}$ DTT $\left(56^{\circ} \mathrm{C} / 550 \mathrm{rpm}\right.$ for 20 minutes), and afterward alkylated by adding $0.5 \mathrm{M}$ IAA and incubated for 20 minutes at room temperature protected from light. Tryptic digestion was carried out at $37^{\circ} \mathrm{C} / 550 \mathrm{rpm}$ for 3 hours, and the samples were separated immediately on a C18 column (AdvanceBio Peptide Mapping, Agilent; $2.1 \times 250 \mathrm{~mm}$ ). The measurements were conducted using a Agilent 6550 iFunnel QTOF mass spectrometer connected to an Agilent 1290 Infinity II HPLC instrument.

Data were analyzed by calculating the peak area of the analyte and the internal standard using Agilent MassHunter Quant software. A standard curve was created by Agilent MassHunter Quant, with which the average ratio of the analyte response to the internal standard response against concentration was plotted.

\section{Complement activation and complement receptors on ECs}

Flow cytometry. Primary HUVECs were cultured on 24-well plates as previously described $(12,54)$ and exposed to heme or RBC MVs at the indicated doses for 30 minutes at $37^{\circ} \mathrm{C}$ in serum-free $\mathrm{M} 199$ cell culture medium (Gibco) in presence or absence of $\mathrm{Hx}$ or HSA. For evaluation of complement activation, HUVECs were washed and further exposed to NHS diluted to 33\% in M199 medium or serum was added to final dilution of $33 \%$ to the wells, already containing heme with and without $\mathrm{Hx}$ for 30 minutes at $37^{\circ} \mathrm{C}$. HUVECs were used for experiments until passage 4 . Cells were washed, detached, labeled, and fixed in $0.5 \%$ formaldehyde. Cells were analyzed by flow cytometry (BD LSR II) and further analyzed by FlowJo X. In addition to the corresponding isotype controls, the following antibodies were used for staining: anti-human MCP-RPE (Bio-Rad, MCA2113PE), anti-human C3aR-PE (BioLegend, 345804), and anti-human C5aR-APC (BioLegend, 344310). After incubation with serum, staining was performed with anti-C3c mouse monoclonal IgG1 antibody (Quidel, A205) or with anti-C5b-9 mouse monoclonal IgG1 (provided by Paul Morgan, Cardiff University, Cardiff, United Kingdom) or an isotype control and revealed by anti-mouse-IgG1-PE secondary antibody (Beckman Coulter, IM0551).

Immunofluorescence. HUVECs were cultured on slides on 24-well plates. Cells were incubated for 5 minutes in presence of $50 \mu \mathrm{M}$ of heme, with or without $5 \mu \mathrm{M}$ of $\mathrm{Hx}$. Cells were fixed in $4 \%$ paraformaldehyde after staining with anti-human P selectin primary antibody (Bio-Rad, MCA796) or an isotype control and revealed with AF-488 secondary antibody (Invitrogen, A21204) and DAPI (to stain nuclei). Staining was analyzed by an Axiovert 200 microscope (Zeiss).

\section{Complement activation in serum}

MVs, generated from HD or SCD patient RBCs, as well as artificial MLVs loaded or not with heme were incubated with buffer, $\mathrm{Hx}$, or HSA for 10 minutes, followed by $33 \% \mathrm{NHS}$ over 30 minutes at $37^{\circ} \mathrm{C}$. MVs were diluted at a final concentration of $1,000 \mathrm{MV} / \mu 1,2,500 \mathrm{MV} / \mu 1$, or 5,000 MV/ $\mu 1$ in TBS with $4 \mathrm{mM}$ $\mathrm{CaCl}_{2}$ and $4 \mathrm{mM} \mathrm{MgCl}_{2}$, allowing activation of the 3 complement pathways. Otherwise, MVs were diluted 
in TBS with $10 \mathrm{mM}$ EGTA and $4 \mathrm{mM} \mathrm{MgCl}_{2}$, thus allowing activation of the AP only. The contribution of the AP was determined by the difference in the signal between the two conditions. $\mathrm{Ba}$ and sC5b-9 levels in the serum were evaluated according to the ELISA kit instructions (Quidel). EDTA was used as a negative control to block complement activation.

\section{Statistics}

Results were analyzed using a statistical software package (GraphPad Prism 5) using the Mann-Whitney test, Kruskal-Wallis with Dunn's test for multiple pairwise comparisons, 2-way ANOVA with Sidak's test for multiple comparisons or Tukey's test for multiple comparisons, or Wilcoxon test after a Shapiro-Wilk test for normality. $P$ values inferior to 0.05 were considered significant.

\section{Study approval}

All animal experiments were conducted with the approval of and in accordance with the recommendations for the care and use of laboratory animals provided by Charles Darwin ethical committee (Paris, France) and with the approval of the French Ministry of Agriculture, Paris, France (APAFIS 3764201601121739330v3). Patients provided written informed consent for the biopsies, the use of clinical data, and the secondary use of histological material for research. This study was performed in accordance with the Declaration of Helsinki. The samples from the HEMIR collection were used for this study. The HEMIR collection was declared to the French Research Ministry (Paris) with approval no. DC.2011-1450.

\section{Author contributions}

LTR and NSM designed the study. NSM, AG, HR, VG, MLF, SK, SB, DC, RN, TRR, TG, MLH, ME, SP, MR, and SC performed research. MF, SLJ, and MR provided care for the patients and provided patient materials. NB and SM provided purified $\mathrm{Hx}$ and instructions for its use. LTR, OPBB, JDD, VFB, NSM, AG, VG, MF, SLJ, ET, PH, SC, and MR discussed the data. NSM, AG, HR, VG, MF, MLF, SK, SB, DC, RN, TRR, MLH, NB, TG, ME, SP, ET, SM, SLJ, PH, SC, MR, JDD, VFB, OPBB, and LTR wrote the manuscript and approved its submission.

\section{Acknowledgments}

This work was supported by grants from Agence Nationale de la Recherche (ANR JCJC-INFLACOMP 2015-2018 ANR-15-CE15-0001 to LTR, ANR JCJC-COBIG ANR-13-JSV1-0006 to JDD, ANR-11IDEX-05-02 Recherche-USPC "HEMIR" to OPBB), by a grant from CSL Behring France to LTR, by CNRS and INSERM. The cytometric and microscopy analyses were performed at the Centre d'Histologie, d'Imagerie et de Cytométrie (CHIC) and the Centre de Recherche des Cordeliers UMRS1138 (Paris, France). We are grateful to the CHIC team for the excellent technical assistance. CHIC is a member of the Université Pierre et Marie Curie Flow Cytometry network (RECYF). We are grateful for excellent technical assistance from the Centre d'Expérimentations Fonctionnelles team of the Centre de Recherche des Cordeliers and for their support with animal experimentation. We thank Elizabeth Huc and the staff of Rodent Breeding Facility Eri970 at Paris-Centre de Recherche Cardiovasculaire for expert mouse breeding and animal care.

Address correspondence to: Lubka T. Roumenina, Cordeliers Research Center, INSERM UMRS 1138; 15 rue de l'Ecole de Medecine, entrance E, fl. 3; 75006 Paris, France. Phone: 33.1.44.27.90.96; Email: lubka.roumenina@crc.jussieu.fr.

1. Dutra FF, Bozza MT. Heme on innate immunity and inflammation. Front Pharmacol. 2014;5:115.

2. Roumenina LT, Rayes J, Lacroix-Desmazes S, Dimitrov JD. Heme: Modulator of plasma systems in hemolytic diseases. Trends Mol Med. 2016;22(3):200-213.

3. Nath KA, Hebbel RP. Sickle cell disease: renal manifestations and mechanisms. Nat Rev Nephrol. 2015;11(3):161-171.

4. Deuel JW, Vallelian F, Schaer CA, Puglia M, Buehler PW, Schaer DJ. Different target specificities of haptoglobin and hemopexin define a sequential protection system against vascular hemoglobin toxicity. Free Radic Biol Med. 2015;89:931-943.

5. Camus SM, et al. Circulating cell membrane microparticles transfer heme to endothelial cells and trigger vasoocclusions in sickle cell disease. Blood. 2015;125(24):3805-3814.

6. Belcher JD, et al. Heme triggers TLR4 signaling leading to endothelial cell activation and vaso-occlusion in murine sickle cell disease. Blood. 2014;123(3):377-390. 
7. Camus SM, et al. Erythrocyte microparticles can induce kidney vaso-occlusions in a murine model of sickle cell disease. Blood. 2012;120(25):5050-5058.

8. Belcher JD, Marker PH, Weber JP, Hebbel RP, Vercellotti GM. Activated monocytes in sickle cell disease: potential role in the activation of vascular endothelium and vaso-occlusion. Blood. 2000;96(7):2451-2459.

9. Merle NS, Church SE, Fremeaux-Bacchi V, Roumenina LT. Complement system part I - Molecular mechanisms of activation and regulation. Front Immunol. 2015;6:262.

10. Merle NS, Noe R, Halbwachs-Mecarelli L, Fremeaux-Bacchi V, Roumenina LT. Complement system part II: Role in immunity. Front Immunol. 2015;6:257.

11. Pawluczkowycz AW, Lindorfer MA, Waitumbi JN, Taylor RP. Hematin promotes complement alternative pathway-mediated deposition of $\mathrm{C} 3$ activation fragments on human erythrocytes: potential implications for the pathogenesis of anemia in malaria. J Immunol. 2007;179(8):5543-5552.

12. Frimat $\mathrm{M}$, et al. Complement activation by heme as a secondary hit for atypical hemolytic uremic syndrome. Blood. 2013;122(2):282-292.

13. Chudwin DS, Papierniak C, Lint TF, Korenblit AD. Activation of the alternative complement pathway by red blood cells from patients with sickle cell disease. Clin Immunol Immunopathol. 1994;71(2):199-202.

14. Mold C, Tamerius JD, Phillips G. Complement activation during painful crisis in sickle cell anemia. Clin Immunol Immunopathol. 1995;76(3 Pt 1):314-320.

15. Chapin J, Terry HS, Kleinert D, Laurence J. The role of complement activation in thrombosis and hemolytic anemias. Transfus Apher Sci. 2016;54(2):191-198.

16. Wang RH, Phillips G, Medof ME, Mold C. Activation of the alternative complement pathway by exposure of phosphatidylethanolamine and phosphatidylserine on erythrocytes from sickle cell disease patients. J Clin Invest. 1993;92(3):1326-1335.

17. De Paepe ME, Trudel M. The transgenic SAD mouse: a model of human sickle cell glomerulopathy. Kidney Int. 1994;46(5):1337-1345.

18. Strauss J, Pardo V, Koss MN, Griswold W, McIntosh RM. Nephropathy associated with sickle cell anemia: an autologous immune complex nephritis. I. Studies on nature of glomerular-bound antibody and antigen identification in a patient with sickle cell disease and immune deposit glomerulonephritis. Am J Med. 1975;58(3):382-387.

19. Pardo V, Strauss J, Kramer H, Ozawa T, McIntosh RM. Nephropathy associated with sickle cell anemia: an autologous immune complex nephritis. II. Clinicopathologic study of seven patients. Am J Med. 1975;59(5):650-659.

20. Smith A, Morgan WT. Haem transport to the liver by haemopexin. Receptor-mediated uptake with recycling of the protein. Biochem J. 1979;182(1):47-54.

21. Sethi S, Fervenza FC. Membranoproliferative glomerulonephritis--a new look at an old entity. NEngl J Med. 2012;366(12):1119-1131.

22. Thurman JM, et al. Detection of complement activation using monoclonal antibodies against C3d. J Clin Invest 2013;123(5):2218-2230.

23. Saraf SL, et al. Haemoglobinuria is associated with chronic kidney disease and its progression in patients with sickle cell anaemia. Br J Haematol. 2014;164(5):729-739.

24. Baek JH, et al. Hemoglobin-driven pathophysiology is an in vivo consequence of the red blood cell storage lesion that can be attenuated in guinea pigs by haptoglobin therapy. J Clin Invest. 2012;122(4):1444-1458.

25. Dimitrov JD, Planchais C, Roumenina LT, Vassilev TL, Kaveri SV, Lacroix-Desmazes S. Antibody polyreactivity in health and disease: statu variabilis. J Immunol. 2013;191(3):993-999.

26. Dimitrov JD, et al. Antibodies use heme as a cofactor to extend their pathogen elimination activity and to acquire new effector functions. J Biol Chem. 2007;282(37):26696-26706.

27. Alaarg A, Schiffelers RM, van Solinge WW, van Wijk R. Red blood cell vesiculation in hereditary hemolytic anemia. Front Physiol. 2013;4:365.

28. van Tits LJ, et al. Plasma annexin A5 and microparticle phosphatidylserine levels are elevated in sickle cell disease and increase further during painful crisis. Biochem Biophys Res Commun. 2009;390(1):161-164.

29. van Beers EJ, et al. Circulating erythrocyte-derived microparticles are associated with coagulation activation in sickle cell disease. Haematologica. 2009;94(11):1513-1519.

30. Schroit AJ, Madsen JW, Tanaka Y. In vivo recognition and clearance of red blood cells containing phosphatidylserine in their plasma membranes. J Biol Chem. 1985;260(8):5131-5138.

31. Morigi M, et al. Alternative pathway activation of complement by Shiga toxin promotes exuberant C3a formation that triggers microvascular thrombosis. J Immunol. 2011;187(1):172-180.

32. Del Conde I, Crúz MA, Zhang H, López JA, Afshar-Kharghan V. Platelet activation leads to activation and propagation of the complement system. J Exp Med. 2005;201(6):871-879.

33. Telen MJ, et al. Randomized phase 2 study of GMI-1070 in SCD: reduction in time to resolution of vaso-occlusive events and decreased opioid use. Blood. 2015;125(17):2656-2664

34. Ataga KI, et al. Crizanlizumab for the prevention of pain crises in sickle cell disease. N Engl J Med. 2017;376(5):429-439.

35. Deuel JW, et al. Hemoglobinuria-related acute kidney injury is driven by intrarenal oxidative reactions triggering a heme toxicity response. Cell Death Dis. 2016;7:e2064.

36. Ingoglia G, et al. Hemopexin counteracts systolic dysfunction induced by heme-driven oxidative stress. Free Radic Biol Med. 2017;108:452-464.

37. Vinchi F, et al. Hemopexin therapy improves cardiovascular function by preventing heme-induced endothelial toxicity in mouse models of hemolytic diseases. Circulation. 2013;127(12):1317-1329.

38. Vinchi F, et al. Hemopexin therapy reverts heme-induced proinflammatory phenotypic switching of macrophages in a mouse model of sickle cell disease. Blood. 2016;127(4):473-486.

39. Graw JA, et al. Haptoglobin or hemopexin therapy prevents acute adverse effects of resuscitation after prolonged storage of red cells. Circulation. 2016;134(13):945-960.

40. Vercellotti GM, et al. Hepatic overexpression of hemopexin inhibits inflammation and vascular stasis in murine models of sickle cell disease. $\mathrm{Mol}$ Med. 2016;22:437-451. 
41. Tolosano E, et al. Defective recovery and severe renal damage after acute hemolysis in hemopexin-deficient mice. Blood. 1999;94(11):3906-3914.

42. Gouveia Z, et al. Characterization of plasma labile heme in hemolytic conditions. FEBS J. 2017;284(19):3278-3301.

43. Merle NS, et al. Characterization of renal injury and inflammation in an experimental model of intravascular hemolysis. Front Immunol. 2018;9:179.

44. Donadee C, et al. Nitric oxide scavenging by red blood cell microparticles and cell-free hemoglobin as a mechanism for the red cell storage lesion. Circulation. 2011;124(4):465-476.

45. Reiter CD, et al. Cell-free hemoglobin limits nitric oxide bioavailability in sickle-cell disease. Nat Med. 2002;8(12):1383-1389.

46. Rother RP, Bell L, Hillmen P, Gladwin MT. The clinical sequelae of intravascular hemolysis and extracellular plasma hemoglobin: a novel mechanism of human disease. JAMA. 2005;293(13):1653-1662.

47. Kato GJ, Steinberg MH, Gladwin MT. Intravascular hemolysis and the pathophysiology of sickle cell disease. J Clin Invest. 2017;127(3):750-760.

48. Ghosh S, et al. Enhanced renal clearance of heme triggers acute kidney injury in sickle cell disease. Blood. 2017;130(Suppl-1):441

49. Schaid TR, et al. Complement activation in a murine model of sickle cell disease: inhibition of vaso-occlusion by blocking C5 activation. Blood. 2016;128(22):158.

50. Berg A, et al. Complement activation correlates with disease severity and contributes to cytokine responses in Plasmodium falciparum malaria. J Infect Dis. 2015;212(11):1835-1840.

51. Lindorfer MA, et al. Compstatin Cp40 blocks hematin-mediated deposition of C3b fragments on erythrocytes: Implications for treatment of malarial anemia. Clin Immunol. 2016;171:32-35.

52. Dumas G, et al. Eculizumab salvage therapy for delayed hemolysis transfusion reaction in sickle cell disease patients. Blood. 2016;127(8):1062-1064.

53. Ryan TM, Ciavatta DJ, Townes TM. Knockout-transgenic mouse model of sickle cell disease. Science. 1997;278(5339):873-876.

54. Roumenina LT, et al. A prevalent C3 mutation in aHUS patients causes a direct C3 convertase gain of function. Blood. 2012;119(18):4182-4191. 